\title{
Molecular and morphological characterisation of new species in the trapdoor spider genus Aname (Araneae: Mygalomorphae: Nemesiidae) from the Pilbara bioregion of Western Australia
}

\author{
FRANCES S. B. HARVEY ${ }^{1}$, VOLKER W. FRAMENAU ${ }^{2,3,6}$, JANINE M. WOJCIESZEK ${ }^{3,7}$, \\ MICHAEL G. RIX ${ }^{2} \&$ MARK S. HARVEY ${ }^{2,3,4,5}$ \\ ${ }^{I}$ Shenton College, 227 Stubbs Terrace, Shenton Park, Western Australia 6008, Australia \\ ${ }^{2}$ Department of Terrestrial Zoology, Western Australian Museum, Locked Bag 49, Welshpool DC, Western Australia 6986, Australia \\ ${ }^{3}$ School of Animal Biology, University of Western Australia, 35 Stirling Highway, Crawley, Western Australia 6009, Australia \\ ${ }^{4}$ Division of Invertebrate Zoology, American Museum of Natural History, Central Park West at 49th Street, New York, NY 10024-5192, \\ USA; and California Academy of Sciences, Golden Gate Park, San Francisco, CA 94103-3009, USA \\ ${ }^{5}$ Corresponding author.E-mail: mark.harvey@museum.wa.gov.au \\ ${ }^{6}$ Current address: Phoenix Environmental Sciences, 1/511 Wanneroo Road, Balcatta, Western Australia 6021, Australia \\ ${ }^{7}$ Current address: School of Veterinary and Biomedical Sciences, Murdoch University, South Street, Murdoch, Western Australia 6150, \\ Australia
}

\begin{abstract}
A study of selected species in the nemesiid spider genus Aname L. Koch, 1873 from the Pilbara bioregion of Western Australia was undertaken using molecular and morphological techniques. Bayesian and parsimony analyses of mitochondrial sequence data from the Cytochrome c Oxidase subunit I (COI) gene found evidence for four species, confirming our initial morphological examination of adult male specimens. These four species are here described as A. mellosa n. sp., A. aragog n. sp., A. ellenae n. sp. and A. marae n. sp. Only the female of A. mellosa n. sp. is described.
\end{abstract}

Key words: taxonomy, systematics, barcoding, COI, Anamini, Teylini, Teyl, Pseudoteyl

\section{Introduction}

Trapdoor spiders of the family Nemesiidae Simon, 1889 are a dominant component of the Australian grounddwelling spider fauna, with 13 genera currently recognised: Aname L. Koch, 1873, Chenistonia Hogg, 1901, Ixamatus Simon, 1887, Kwonkan Main, 1983, Merredinia Main, 1983, Namea Raven, 1984, Pseudoteyl Main, 1985, Stanwellia Rainbow and Pulleine, 1918, Swolnpes Main and Framenau, 2009, Teyl Main, 1975, Teyloides Main, 1985, Xamiatus Raven, 1981 and Yilgarnia Main, 1986 (Platnick 2012). The most speciose of these genera, Aname, is found across most of Australia and currently contains 33 named species (Platnick 2012), although many more species have been collected and await description (VWF, unpubl. data; B.Y. Main and R.J. Raven, pers. comm.). They can occur in reasonable densities in both mesic and arid habitats, and males are regularly caught in pitfall traps after they emerge from their burrows and wander in search of females during their mating season. Juveniles and adults construct burrows within soil and, whilst many have open holes, some, such as Aname turrigera Main, 1994, from the Nullarbor region of southern Australia, build long silken tubes through Triodia spinifex bushes (Main 1994).

Few species of Aname have been described from Western Australia, and all named species are from the southern half of the state. These include Aname armigera Rainbow and Pulleine, 1918, A. cuspidata (Main, 1954), A. fuscocincta Rainbow and Pulleine, 1918, A. mainae Raven, 2000, A. tepperi (Hogg, 1901), A. turrigera Main, 1994 and A. villosa (Rainbow and Pulleine, 1918) (Hogg 1901; Main 1954, 1985, 1994; Rainbow \& Pulleine 1918; Raven 2000). The status and taxonomic position of some of these species are still unresolved, due largely to lack of modern revisionary taxonomic studies and a lack of adult males for many species (which typically display the best 
diagnostic morphological features). In addition, the generic limits of Aname remain obscure. For example, Raven (1981) synonymised Chenistonia with Aname, but later (Raven 2000) resurrected the genus for his Aname maculata-group (e.g. Raven 1984). The characters used to diagnose this group differ greatly from Main's (1985) concept of Chenistonia. These taxonomic problems are not surprising given the diversity of Nemesiidae within Australia, and given that the limits of the family itself are also poorly understood (Goloboff 1993, 1995; Hedin \& Bond 2006).

The present study documents a small proportion of a diverse assemblage of spiders attributable to the genus Aname from north-western Australia. While species of Aname have been known from the region for many years, there are currently no described species. We employed a molecular barcoding approach (Barrett \& Hebert 2005; Hebert et al. 2003; Prendini 2005) to assist in delimitating species of Aname that were originally identified using the morphology of adult males, focusing primarily on the distinct shape of the male pedipalp and leg I, but also on somatic features such as colouration and setal patterns. Molecular clades and morphological species concepts were concordant, and we here recognise and describe four species, representing the first nemesiid spiders to be named from northern Western Australia.

\section{Material and methods}

Morphology. The specimens examined in this study are lodged at the Western Australian Museum, Perth (WAM) and the Queensland Museum, Brisbane (QM). Specimens used for morphological examination were preserved in $75 \%$ ethanol. Auto-montaged images of males were taken at different focal planes ( $c a$. 20-30 images) with a Leica DFC500 digital camera attached to a Leica MZ16A stereo microscope, using Leica Application Suite (LAS) version 2.5.OR1 software. Images of females were taken using a similar protocol but with different optical instruments (Leica DFC295 camera on a Leica M205C microscope; image processing with LAS v. 3.7.0). Female genitalia were prepared for imaging by submersion in lactic acid for $c a .12 \mathrm{hrs}$. All measurements were taken with an ocular micrometer and are expressed in millimeters, and were based on the methodology proposed by Coyle (1971); however, we measured sternum and labium together.

The "leg formula" is here given as the leg length divided by the carapace length. The "tibial index" is defined as: (dorsal proximal width of patella / length of tibia + patella) x 100. It is a measurement of the 'stoutness' of the legs (Petrunkevitch 1942). Total length is measured dorsally including the chelicerae, but excluding the spinnerets.

Molecular methods. Individual legs of Aname specimens were preserved in 100\% ethanol by the collectors of the specimens, and approximately $2 \mathrm{~mm}^{3}$ of muscle tissue was removed from one leg of each specimen for use in the molecular analyses. Whole genomic DNA was extracted using the Qiagen DNeasy Blood and Tissue Kit (animal tissue protocol). Polymerase chain reaction (PCR) amplification of a $658 \mathrm{bp}$ fragment of the mitochondrial Cytochrome c Oxidase subunit I (COI) gene was achieved using the primers LCO1490 and HCO2198 (Folmer et al. 1994), in an Eppendorf Mastercycler ep gradient $S$ thermal cycler. Each $25 \mu 1$ PCR contained $2 \mu$ l of extracted DNA, $400 \mathrm{nM}$ of the forward primer, $400 \mathrm{nM}$ of the reverse primer, 1 X PCR buffer (Invitrogen), $4.0 \mathrm{mM} \mathrm{MgCl}_{2}$, $200 \mu \mathrm{M}$ of each dNTP (Invitrogen), $1.25 \mathrm{U}$ of Taq DNA polymerase (Invitrogen) and $\mathrm{dH}_{2} \mathrm{O}$. PCR cycling conditions were as follows: $94^{\circ} \mathrm{C}$ for 3 minutes; 30 cycles of $94^{\circ} \mathrm{C}$ for $30 \mathrm{sec}, 48^{\circ} \mathrm{C}$ for $30 \mathrm{sec}, 72^{\circ} \mathrm{C}$ for $2 \mathrm{~min}$; and a final extension of $72^{\circ} \mathrm{C}$ for 2 minutes. To test for contamination, negative control samples were included with each set of PCRs; these samples included $2 \mu \mathrm{l}$ of nuclease-free water instead of template DNA. Amplified DNA samples were purified using the MoBio Ultra-Clean PCR Clean-up Kit. Bi-directional DNA sequencing was performed by Macrogen Corporation (South Korea), using supplied forward and reverse PCR primers. Sequence (.ab1) files for the coding and non-coding strands were assembled automatically as anti-parallel contigs, and visualised as text sequences and chromatograms using Sequencher 4.8 (Demonstration Version). Annotated, length-invariable sequences were saved as text files and imported into ClustalX Version 1.83 (Thompson et al. 1997) for alignment. Over 30 specimens of Aname were sequenced for this study (see Table 1), with each sequenced specimen further denoted by a superscript 'DNA' code in the Material Examined sections. Two species of other nemesiid genera, Pseudoteyl sp. and Teyl luculentus Main, 1975, were also sequenced, and three additional COI sequences were retrieved from GenBank for use as outgroups (Table 1). These outgroup sequences were from the theraphosid Ornithoctonus huwena (Wang, Peng \& Xie, 1993), the diplurid Euagrus chisoseus Gertsch, 1939, and the liphistiid Songthela hangzhouensis (Chen, Zhang and Zhu, 1981). Ornithoctonus huwena, originally described as Selenocos- 
mia huwena by Wang et al. (1993), has since been synonymised with Haplopelma schmidti von Wirth, 1991 by Schmidt (2000) and we here use the currently accepted name (Platnick 2012; Zhu \& Zhang 2008).

Phylogenetic analyses. To infer phylogenetic relationships among species, an unpartitioned Bayesian phylogenetic analysis of the COI mtDNA data was executed using MrBayes Version 3.1.2 (Ronquist \& Huelsenbeck 2003). Prior to analysis, Modeltest3.7 (Posada \& Crandall 1998) was used to choose the appropriate model of nucleotide substitution under an Akaike Information Criterion (AIC) framework. For the COI data, the GTR+I+G model was invoked with the following MrBayes likelihood settings: [Lset nst=6 rates=gamma]. Four Markov Chain Monte Carlo (MCMC) chains were run for 2,000,000 generations, sampling every 1,000 generations, with the first 100,000 sampled trees discarded as 'burnin' ([burnin=100]), as confirmed by visualisation of the log likelihood trace and the average standard deviation of split frequencies being $<0.01$. Posterior probabilities were calculated and reported on a 50\% majority-rule consensus tree of the post-burnin sample.

A parsimony analysis of the COI data was also performed using PAUP* Version 4.0b10 (Swofford 2002). Trees were analysed using a tree-bisection-reconnection (TBR) search algorithm, with 10,000 replicates and 10 trees held at each step. Clade support values were estimated using non-parametric bootstrapping (Felsenstein 1985) in PAUP*, with 1,000 pseudoreplicates of a heuristic (TBR) search algorithm incorporating 10 replicates of random stepwise addition of taxa and 10 trees held at each step.

\section{Results and discussion}

Phylogeny and taxonomy. Based on distinct molecular, genitalic and somatic differences we here recognise four new species in the genus Aname from the Pilbara bioregion of Western Australia. Bayesian analysis using a fragment of the mitochondrial gene $\mathrm{COI}$ revealed four major lineages, three of which were monophyletic and strongly supported (with posterior probabilities $>95 \%$ ) and a fourth was represented by only a single specimen (Fig. 9). These four lineages were congruent with existing morphological species concepts (as described below), providing preliminary evidence for a consistency between barcoding and morphological methods in this group and between phylogenetic and biological species concepts (e.g. De Queiroz 2007; Donoghue 1985; Nixon \& Wheeler 1990). All three monophyletic (multi-specimen) species lineages showed varying levels of internal genetic structure, usually corresponding to locality-specific haplotype clades (Fig. 9, Table 1). The analysis failed, however, to recover Aname as a monophyletic clade, given the poorly supported sister-group relationship inferred for A. marae $\mathbf{n}$. sp. and the two other nemesiids included in the analysis (i.e. Pseudoteyl sp. and Teyl luculentus). Parsimony analysis of the same COI dataset revealed 24 equally parsimonious trees, with a strict consensus tree revealing congruent monophyly of the four major species lineages, each further supported by high (> 85) bootstrap support. As for the Bayesian analysis, inter-specific phylogenetic relationships were poorly resolved and very poorly supported by the parsimony analysis of the $\mathrm{COI}$ dataset.

The inferred non-monophyly of Aname in the current study is not unexpected, given the problems associated with inferring deeper phylogenies using single locus mitochondrial datasets (e.g. Brito \& Edwards 2009; Prendini 2005), and the untested phylogenetic relationship between Teyl, Pseudoteyl and the currently described Aname species. The characterisation of Australian nemesiid genera is still not fully understood, with some genera, including Aname, lacking unequivocal synapomorphies denoting monophyly (Raven 1985b). The molecular analyses of Hedin and Bond (2006) — which incorporated species of Aname as well as many other nemesiids from Australia and elsewhere-found two unidentified species of Aname grouping separately from Aname tepperi (Hogg, 1902), the latter of which paired with Kwonkan and then the Teylini (= Teyl, Namea and Pseudoteyl). Clearly, significant additional systematic study is required to adequately resolve deeper inter-generic and tribal relationships among Australian Nemesiidae, especially in Western Australia where a considerable proportion of the Australian genera and species can be found.

Distribution. Each of the four species described in this study possess unique distribution patterns, with two of the four species currently known from only very restricted ranges. Aname aragog $\mathbf{n}$. sp. has been found at a single location in the eastern Pilbara near Newman (Fig. 6) and A. marae n. sp. occurs at a single location in the central Pilbara near Tom Price (Fig. 8). Similar narrow distribution patterns have been found in other northern Australian species of Aname, with many taxa reported from single localities (Raven 1985b). In many cases this may reflect a sampling bias, although in Western Australia the Pilbara region has been surveyed extensively in recent years either through scientific programs (Durrant et al. 2010; McKenzie et al. 2009) or as part of recent environmental 
assessment studies (see Harvey et al. 2011). Consequently, the distributions of the two common and more broadly distributed species described here are relatively well documented. Indeed, A. mellosa n. sp. appears to be widespread throughout north-western Australia (Fig. 5) and occurs over a wide area of the Pilbara and into the Midwest region of Western Australia. However, deep genetic structuring of COI haplotypes (see Fig. 9) may be indicative of cryptic speciation within the A. mellosa-complex, and additional, more rigorous multi-locus genetic studies are required to determine whether other known populations are conspecific with the Jinayri type specimens. Aname ellenae n. sp. appears to be reasonably widespread in the western Pilbara region, currently known from at least two widely separated localities south-west and east of Dampier (Fig. 7). These two more common species were also reported from the Department of Environment and Conservation (DEC) Pilbara Biological Survey as Aname 'MYG001' (= A. mellosa) and Aname 'MYG100' (= A. ellenae) (Durrant et al. 2010). Overall, the DEC Pilbara survey found 12 putative Aname species and therefore a similar diversity as was recovered during the earlier DEC/ WAM survey of the Carnarvon Basin bioregion south-west of the Pilbara (13 species) (Main et al. 2000). Interestingly, neither study reported A. aragog or A. marae, suggesting that these surveys-conducted primarily using pitfall traps-may not have sufficiently documented the north-western Australian Aname fauna, which may include a number of additional short-range endemic species (Harvey 2002) yet to be discovered.

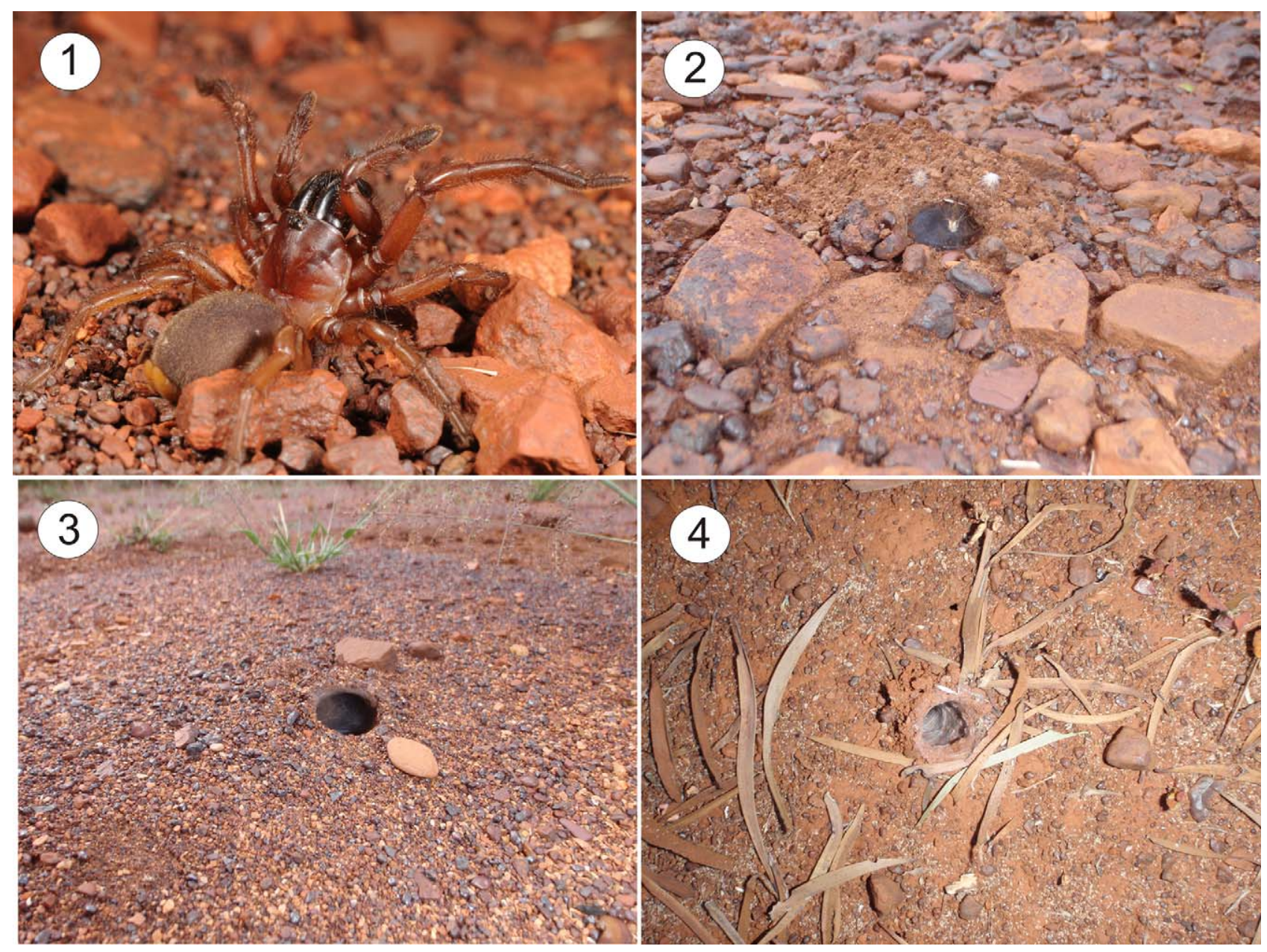

FIGURES 1-4. Aname mellosa n. sp.: 1, living female (WAM T113612); 2-4, burrow entrances: 2, female (WAM T113574); 3, female (WAM T113576); 4, female (WAM T113582). Images courtesy of R. Teale. 


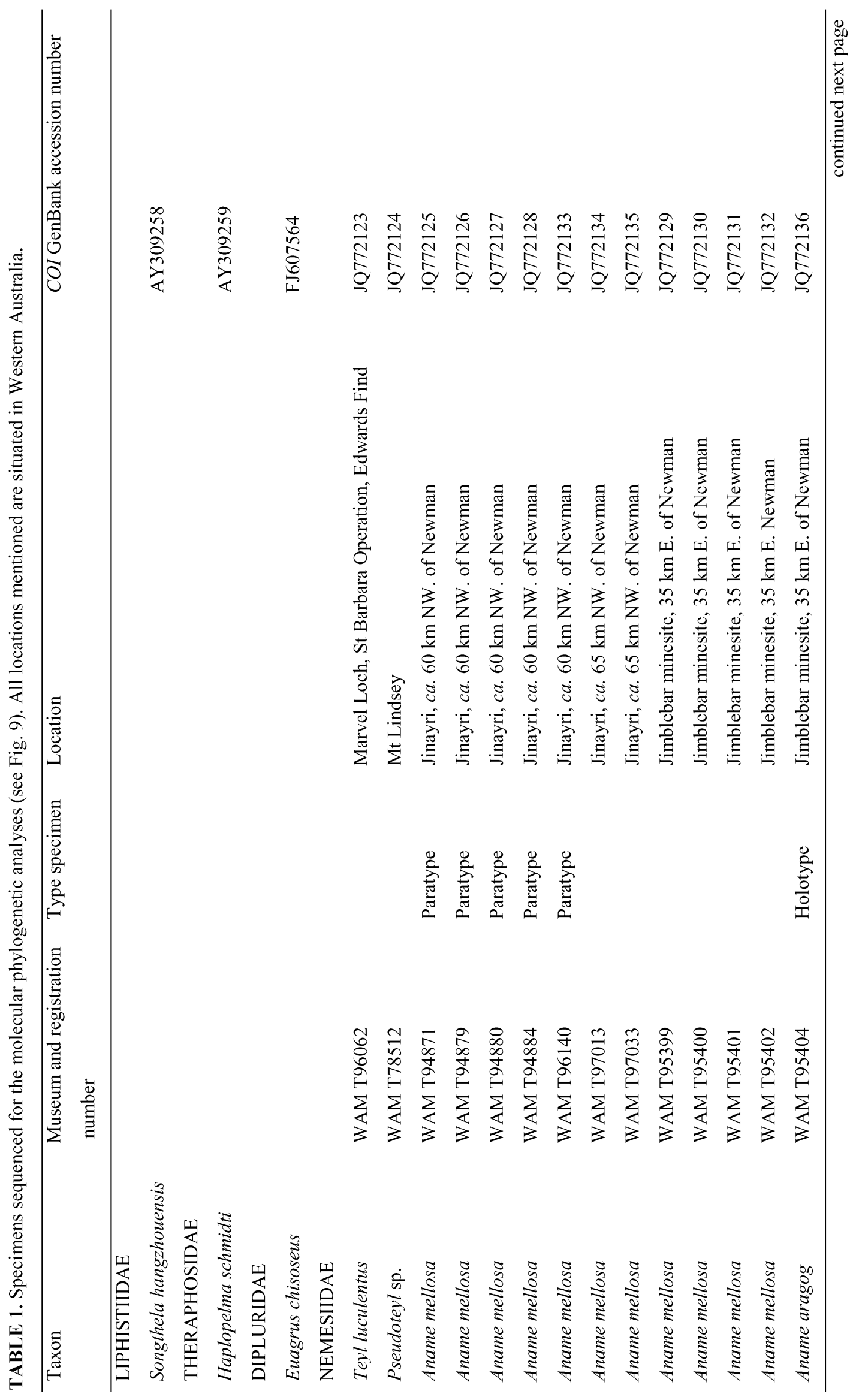




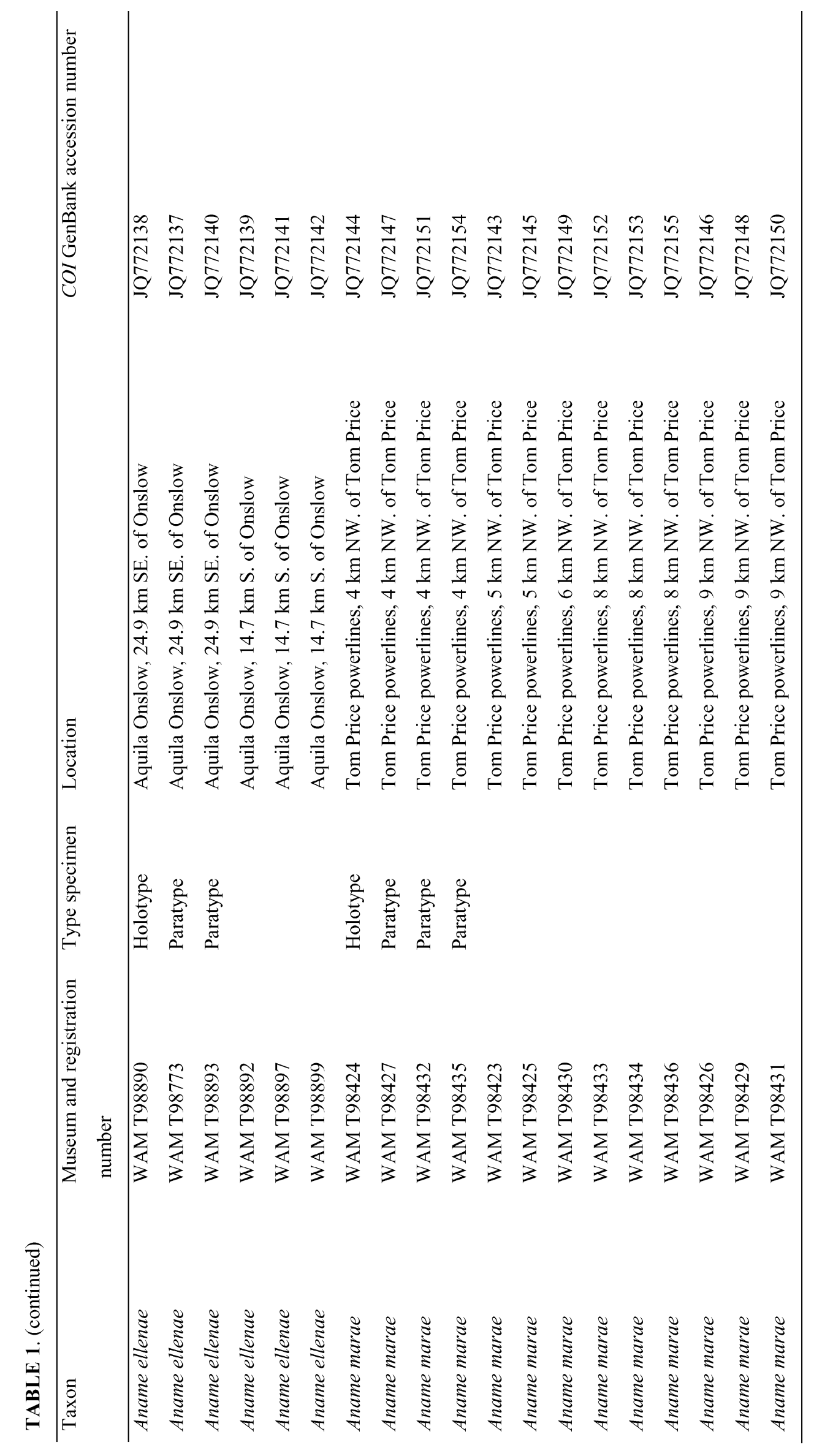



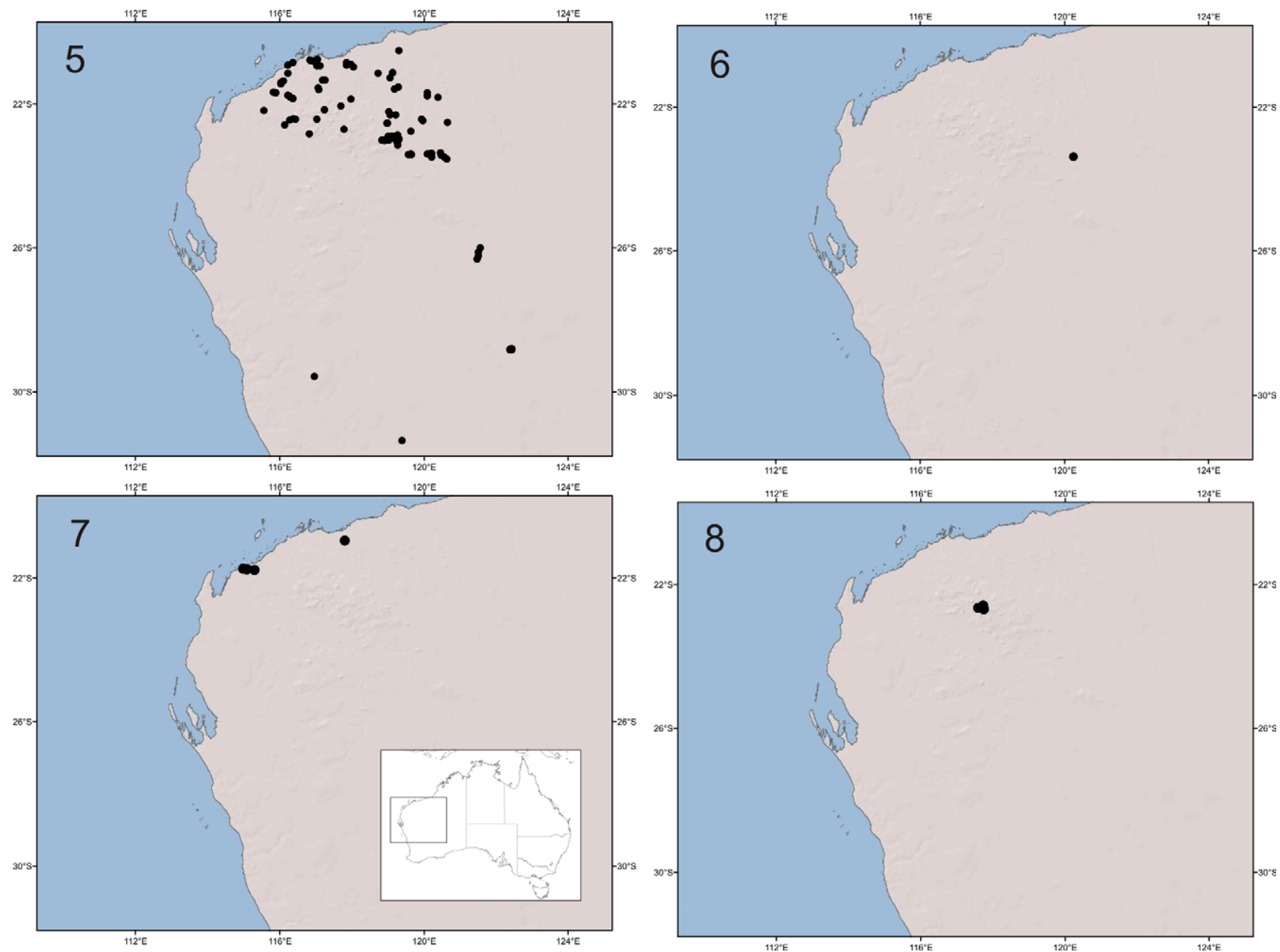

FIGURES 5-8. Maps showing collecting locations of Aname species in Western Australia: 5, A. mellosa n. sp.; 6, A. aragog n. sp.; 7, A. ellenae n. sp.; 8, A. marae n. sp.

\section{Systematics}

\section{Family Nemesiidae Simon, 1889}

\section{Subfamily Anaminae Simon, 1889}

\section{Genus Aname L. Koch, 1873}

Aname L. Koch 1873: 465. Type species Aname pallida L. Koch, 1873, by monotypy.

Dekana Hogg 1902: 138 (synonymised by Raven 1981: 328 and Main 1982b: 27). Type species Dekana diversicolor Hogg, 1902 , by original designation.

Proshermacha Simon 1908: 113 (synonymised with Chenistonia by Main 1982a: 113). Type species Proshermacha subarmata Simon, 1908 (junior synonym of Chenistonia teppperi Hogg, 1902), by subsequent designation of Rainbow (1911).

Sungenia Rainbow and Pulleine 1918: 162 (synonymised by Raven 1981: 328 and Main 1982b: 27). Type species Chenistonia (Dekana) atra Strand, 1913, by monotypy.

Dolichosternum Rainbow and Pulleine 1918: 168 (synonymised by Raven 1981: 328). Type species Dolichosternum attenuatum Rainbow and Pulleine, 1918 (junior synonym of Ixamatus distinctus Rainbow, 1914), by monotypy.

Diagnosis. Species of Aname differ from all other nemesiids by the presence of a large mega-spur on the tibia of leg I generally in a mid-distal position (e.g. Fig. 15), the absence of spines on the pedal tarsi (e.g. Fig. 19), and the absence of cuspules on the pedal coxae (e.g. Fig. 12) (following Main 1982b, 1983, 1986; Raven 1981). The male pedipalpal bulb is spherical to pear-shaped, with the embolus originating from the bulb in a distal position (e.g. Figs 17-20); the shape of the embolus is usually long and slender, and slightly curved in profile (e.g. Figs 17-20). 
Remarks. While we are confident that there are four distinct species represented in our samples, we found considerable difficulty in distinguishing these species from some previously named species. Differentiating our species from those for which males have been described was not difficult. Several species of Aname are currently known only from adult females and for which spermathecae have not been described or illustrated. These are: A. armigera Rainbow \& Pulleine, 1918 (from south-western Australia), A. aurea Rainbow \& Pulleine, 1918 (from New South Wales), A. coenosa Rainbow \& Pulleine, 1918 (from South Australia), A. comosa Rainbow \& Pulleine, 1918 (from South Australia), A. cuspidata (Main, 1954) (from south-western Australia), A. fuscocincta Rainbow \& Pulleine, 1918 (from south-western Australia), A. grandis Rainbow \& Pulleine, 1918 (from South Australia), A. hirsuta Rainbow \& Pulleine, 1918 (from South Australia), A. maculata (Rainbow \& Pulleine, 1918) (from south-western Australia), A. platypus (L. Koch, 1875) (possibly from Australia), A. tasmanica Hogg, 1902 (from Tasmania) and A. villosa (Rainbow \& Pulleine, 1918) (from south-western Australia) (Hogg 1902; Koch 1875; Main 1954; Rainbow \& Pulleine 1918). As the type localities of all of these species are located at least 1,000 km away from the Pilbara region, we are confident that we have not inadvertently described a species that will eventually become a junior synonym.

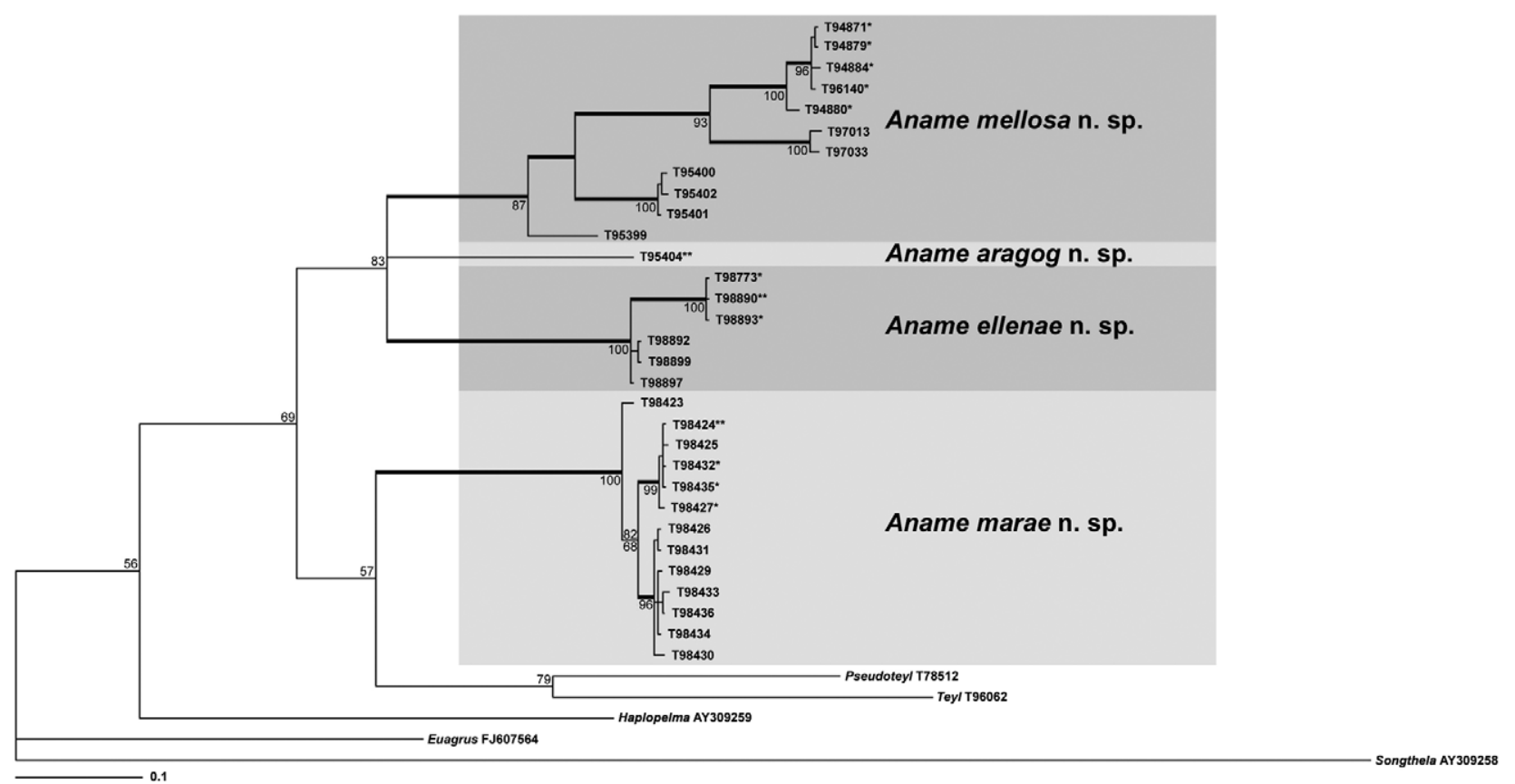

FIGURE 9. Bayesian majority-rule consensus tree, showing results from the phylogenetic analyses of the COI mtDNA dataset (658 bp, 36 taxa). Thickened branches represent clades with $>95 \%$ posterior probability (PP) support, with lower PP values shown above other nodes. Numbers below nodes denote bootstrap support values following parsimony analysis of the same dataset. Asterisks denote holotype $(* *)$ and paratype $(*)$ specimens, respectively.

Aname mellosa n. sp.

Figs 1-5, 9-24

Type material. AUSTRALIA: Western Australia: holotype male, Jinayri, ca. $60 \mathrm{~km}$ NW. of Newman, 22 58'05.7'S, $119^{\circ} 17^{\prime} 06.1$ 'E, 3 February 2009, pitfall trap, J. Gollan, N. Sullivan, M. Semeniuk, M. Beatson (WAM

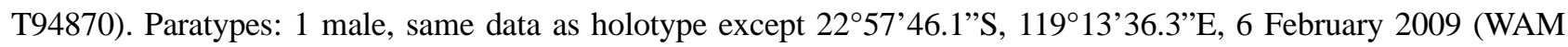
T96116); 2 males, same data except 22 57'46.5”S, 119¹3'36.5”E, 7 February 2009 (WAM T96121, T94864); 1

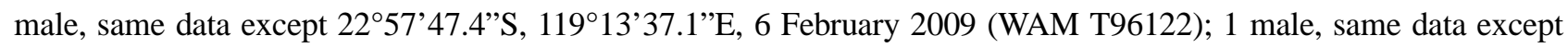

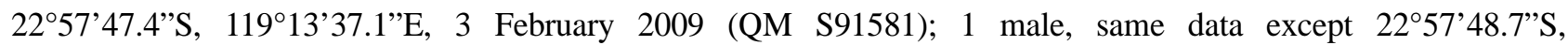

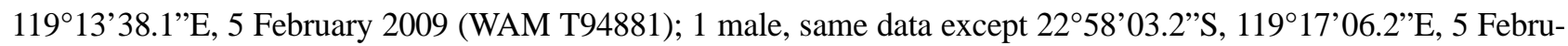

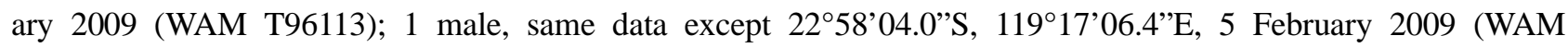
T94871 ${ }^{\mathrm{DNA}}$ ); 1 male, same data except $22^{\circ} 58^{\prime} 05.0^{\prime \prime} \mathrm{S}, 119^{\circ} 17^{\prime} 06.2^{\prime \prime} \mathrm{E}, 5$ February 2009 (WAM T96117); 2 males, same data except $22^{\circ} 58^{\prime} 09.5^{\prime}$ 'S, $119^{\circ} 15^{\prime} 35.1^{\prime \prime}$ 'E, 3 February 2009 (WAM T94885, T94872); 1 male, same data except 22 58'09.5”S, 119¹5'36.2”E, 7 February 2009 (WAM T96128); 2 males, same data except 22 58'09.5”S, $119^{\circ} 15^{\prime} 36.2^{\prime \prime} \mathrm{E}, 1$ February 2009 (WAM T94884 ${ }^{\mathrm{DNA}}$, T94882); 1 male, same data except 22 $58^{\circ}$ '09.5”'S, $119^{\circ} 15^{\prime} 36.4^{\prime \prime} \mathrm{E}$, 
5 February 2009 (WAM T94857); 1 male, same data except 2258’09.5”S, 119¹5’36.4”E, 6 February 2009 (WAM T94862); 2 males, same data except $22^{\circ} 58^{\prime} 09.8^{\prime \prime} \mathrm{S}, 119^{\circ} 15^{\prime} 36.5^{\prime} \mathrm{E}, 7$ February 2009 (WAM T96144, T96126); 1 male, same data except $22^{\circ} 58^{\prime} 10.2^{\prime \prime} \mathrm{S}, 119^{\circ} 15^{\prime} 37.1$ ”E, 6 February 2009 (WAM T94863); 1 male, same data except

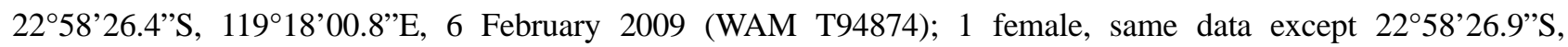
$119^{\circ} 18^{\prime} 03.1$ 'E, 1 February 2009 (WAM T96145); 2 males, same data except 22 $58^{\prime} 26.9^{\prime \prime}$ 'S, $119^{\circ} 18^{\prime} 03.1$ 'E, 6 February 2009 (WAM T94856, T94860); 1 male, same data except 22 $2^{\circ} 58^{\prime} 27.0^{\prime \prime} \mathrm{S}, 119^{\circ} 18^{\prime} 04.0^{\prime \prime} \mathrm{E}, 7$ February 2009 (WAM T94886); 1 male, same data except 22058'42.6”S, 119¹5’39.8”E, 5 February 2009 (WAM T96129); 1 male, same data except $22^{\circ} 58^{\prime} 42.9^{\prime \prime} \mathrm{S}, 119^{\circ} 15^{\prime} 41.2^{\prime \prime} \mathrm{E}, 7$ February 2009 (WAM T94859); 1 male, same data except 22 $58^{\prime} 43.5^{\prime \prime} \mathrm{S}$,

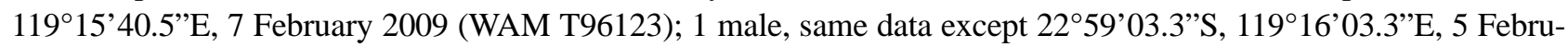
ary 2009 (WAM T96114); 1 male, same data except $22^{\circ} 59^{\prime} 03.7^{\prime \prime}$,, $19^{\circ} 18^{\prime} 03.8^{\prime \prime}$ E, 2 February 2009 (WAM T94879 ${ }^{\mathrm{DNA}}$ ); 1 male, same data except $22^{\circ} 59^{\prime} 04.5^{\prime}$ 'S, $119^{\circ} 16^{\prime} 02.3$ ”'E, 7 February 2009 (WAM T94866); 1 male, same data except $22^{\circ} 59^{\prime} 04.5^{\prime \prime} \mathrm{S}, 19^{\circ} 16^{\prime} 02.3^{\prime \prime} \mathrm{E}, 6$ February 2009 (WAM T94868); 2 males, same data except $22^{\circ} 59^{\prime} 08.6^{\prime \prime} \mathrm{S}, 119^{\circ} 15^{\prime} 02.8^{\prime \prime} \mathrm{E}, 7$ February 2009 (WAM T94854, T94861); 1 male, same data except 2259'08.8”S,

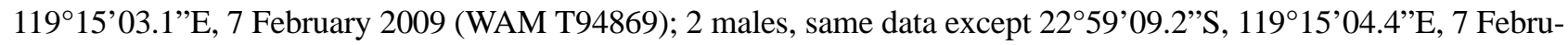
ary 2009 (WAM T94853, T96118); 1 male, same data except 2259'09.5”'S, 119¹5’35.1”'E, 7 February 2009 (WAM T94867); 1 male, same data except 22 $59^{\prime} 14.1^{\prime \prime S}, 119^{\circ} 16^{\prime} 09.6$ 'E, 6 February 2009 (WAM T94858); 1 male, same

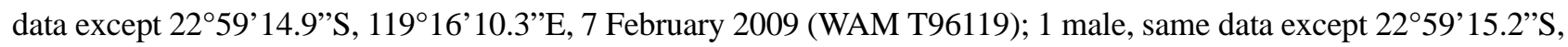

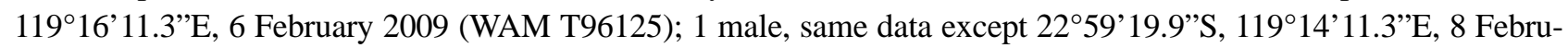
ary 2009 (WAM T96127); 1 male, same data except 22 59'21.3”'S, 119¹4'10.7”'E, 6 February 2009 (WAM T94865); 1 male, same data except $22^{\circ} 59^{\prime} 23.1^{\prime \prime}$ S, 119 $14^{\circ} 11.1$ ”E, 8 February 2009 (WAM T94851); 1 male, same data except

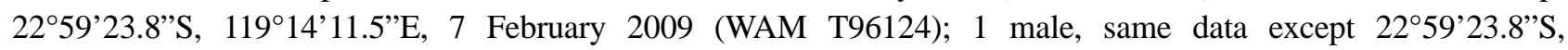

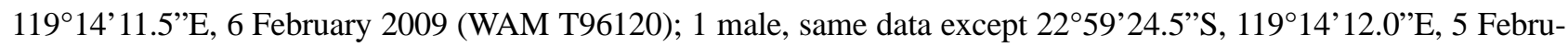
ary 2009 (WAM T94883); 1 male, same data except 2300'23.3”'S, 119¹4'42.6”'E, 7 February 2009 (WAM T94876); 1 male, same data except $23^{\circ} 00^{\prime} 23.9^{\prime \prime}$ S, 119 $19^{\circ} 43.6$ "E, 5 February 2009 (WAM T96151); 1 male, same data except

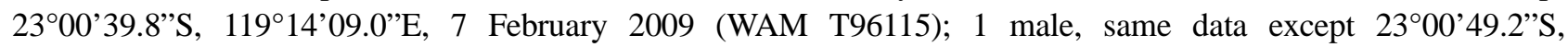

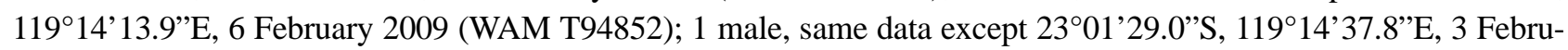

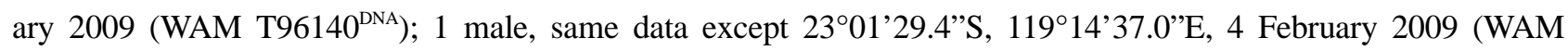

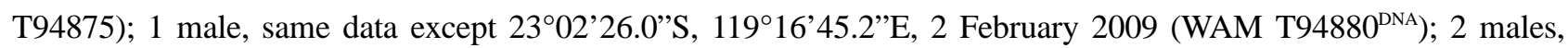
same data except $23^{\circ} 03^{\prime} 08.8^{\prime \prime} \mathrm{S}, 119^{\circ} 16^{\prime} 14.9^{\prime \prime}$, 3 February 2009 (WAM T94873, T94878); 1 male, same data except $23^{\circ} 03^{\prime} 09.8^{\prime \prime} \mathrm{S}, 119^{\circ} 16^{\prime} 13.9^{\prime \prime} \mathrm{E}, 5$ February 2009 (WAM T94850); 1 female, same data as holotype except 2259'24.5”'S, 119¹4'12.0”'E, 8 February 2009 (WAM T96150).

Other material examined. AUSTRALIA: Western Australia: 1 male, Abydos mine lease, $c a .64 \mathrm{~km} \mathrm{~W}$. of

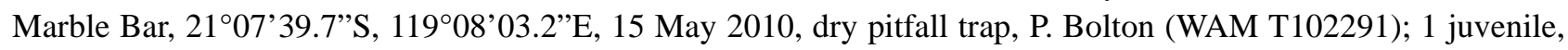
same data except $21^{\circ} 07^{\prime} 50.2^{\prime \prime S}, 1^{\circ} 9^{\circ} 07^{\prime} 32.7^{\prime \prime} \mathrm{E}, 14$ May 2010 (WAM T102290); 1 male, same data except

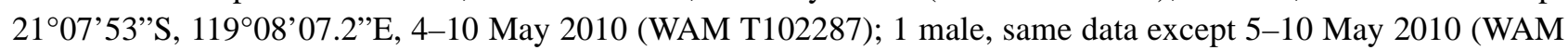
T102288); 1 male, same data except $21^{\circ} 07^{\prime} 54.9^{\prime \prime} \mathrm{S}, 119^{\circ} 07^{\prime} 54^{\prime \prime} \mathrm{E}, 28-5$ May 2010, wet pitfall trap (WAM T102284); 1 male, API Hardey, 96.9 km WNW. of Paraburdoo, 22 49'04”'S, 116 48'53”E, 24 March 2010, dug from burrow, P. Runham (WAM T110361); 1 male, Aquila, $12.75 \mathrm{~km} \mathrm{SE}$. of Karratha, 2048'34”S, 116 56'29'E, 8 April 2009, dug from burrow, P. Runham (WAM T100079); 1 male, same data except 5 April 2009 (WAM T100080); 1 male, Aquila, $20.6 \mathrm{~km}$ E. of Karratha, 20 $45^{\prime} 45^{\prime \prime} \mathrm{S}, 117^{\circ} 02^{\prime} 31^{\prime \prime} \mathrm{E}, 5$ April 2009, dug from burrow, P. Runham (WAM T100077); 1 male, same data except 7 April 2009 (WAM T100078); 1 male, Area C, $101 \mathrm{~km} \mathrm{NW.}$

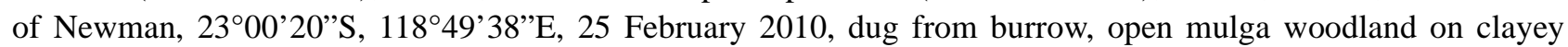
loam plain, M. Greenham (WAM T101158); 1 female, same data except D. Kamien, T. Sachse (WAM T101162); 1 male, Area C, $61.8 \mathrm{~km}$ NW. of Newman, 22 $59^{\prime} 55^{\prime}$ 'S, $118^{\circ} 52^{\prime} 07^{\prime} \mathrm{E}, 1$ May 2010, wet pit trap, S. Ford, G. O'Con-

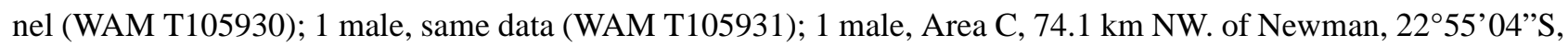
119¹1’21'E, 1 May 2010, wet pit trap, S. Ford, G. O’Connel (WAM T105957); 1 female, same data except $22^{\circ} 55^{\prime} 05^{\prime}$ 'S, $119^{\circ} 11^{\prime} 21^{\prime \prime E}, 18$ February 2010 , dug from burrow, drainage in valley/flats, D. Kamien (WAM T101163); 1 female, Area C, 75.7 km NW. of Newman, 2254'21”S, 119¹0'48”E, 19 February 2010, dug from burrow, loamy plain, D. Kamien, T. Sachse (WAM T101165); 1 male, Area C, $75.8 \mathrm{~km} \mathrm{NW}$. of Newman, 22 $2^{\circ} 4^{\prime} 21^{\prime \prime}$ S, $119^{\circ} 10^{\prime} 48^{\prime \prime}$ E, 1 May 2010, wet pit trap, S. Ford, G. O'Connel (WAM T105943); 1 male, Area C, 78.9

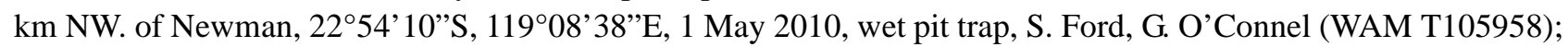
1 male, Area C, 81.4 km NW. of Newman, 2257'29’S, 11904'09”'E, 1 May 2010, wet pit trap, S. Ford, G. O'Con- 
nel (WAM T105914); 1 male, Area C, 81.8 km NW. of Newman, 2300'20”S, 11901'58”E, 21 February 2010, dug from burrow, plateau/top of rocky hill, M. Greenham (WAM T101156); 1 female, Area C, $82.6 \mathrm{~km} \mathrm{NW}$. of Newman, 22 ${ }^{\circ} 58^{\prime} 54^{\prime \prime}$ S, 11900'49”'E, 20 February 2010, dug from burrow, Gully, Z. Hamilton (WAM T101161); 1 male, same data except $22^{\circ} 58^{\prime} 55^{\prime}$ 'S, $119^{\circ} 00^{\prime} 50^{\prime \prime} \mathrm{E}, 1$ May 2010, wet pit trap, S. Ford, G. O'Connel (WAM

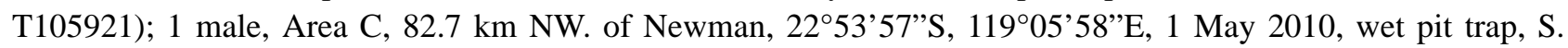
Ford, G. O'Connel (WAM T105954); 1 male, Area C, 82.7 km NW. of Newman, 2257'49’'S, 11902'50'E, 1 May 2010, wet pit trap, S. Ford, G. O'Connel (WAM T105910); 1 female, Area C, $82.7 \mathrm{~km} \mathrm{NW.} \mathrm{of} \mathrm{Newman,}$ $22^{\circ} 57^{\prime} 53^{\prime \prime S}, 119^{\circ} 02^{\prime} 53^{\prime}$ 'E, 20 February 2010, dug from burrow, mulga plain, M. Greenham (WAM T101164); 1 male, Area C, $82.7 \mathrm{~km}$ NW. of Newman, $2^{\circ} 00^{\prime} 18^{\prime \prime} \mathrm{S}, 11^{\circ} 01^{\prime} 26^{\prime \prime} \mathrm{E}, 1$ May 2010, wet pit trap, S. Ford, G. O'Con-

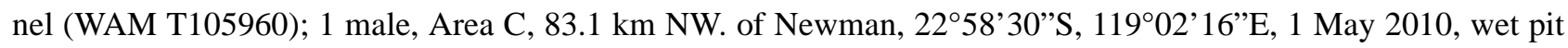
trap, S. Ford, G. O'Connel (WAM T105956); 1 male, same data (WAM T105967); 1 male, Area C, 83.9 km NW. of Newman, 22 59'35”S, 11900'57’E, 1 May 2010, wet pit trap, S. Ford, G. O'Connel (WAM T105932); 1 male,

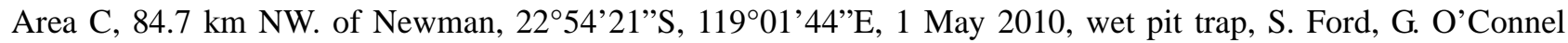

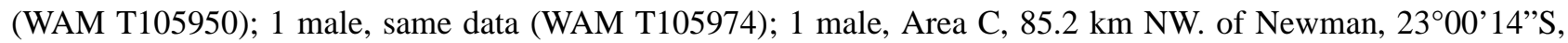
$118^{\circ} 59^{\prime} 41^{\prime}$ 'E, 22 February 2010, dug from burrow, Z. Hamilton (WAM T101154); 1 male, Area C, $86.2 \mathrm{~km} \mathrm{NW.} \mathrm{of}$ Newman, 2300'05”S, 118 58'54”E, 1 May 2010, wet pit trap, S. Ford, G. O'Connel (WAM T105928); 1 male,

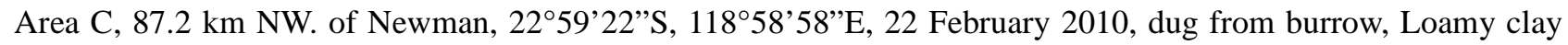
plain, D. Kamien (WAM T101155); 1 male, Area C, 89 km NW. of Newman, 2257’23”S, 11859’00”E, 1 May 2010, wet pit trap, S. Ford, G. O'Connel (WAM T105911); 1 male, same data (WAM T105912); 1 male, same data (WAM T105975); 1 male, Area C, 89.2 km NW. of Newman, 2257'56”S, 118 58'32”E, 23 February 2010, dug from burrow, rocky loam plain, D. Kamien, T.Sachse (WAM T101157); 1 male, Area C, 89.4 km NW. of Newman, 22 $2^{\circ} 8^{\prime} 49^{\prime \prime}$ 'S $118^{\circ} 57^{\prime} 44^{\prime \prime}$ E, 1 May 2010, wet pit trap, S. Ford, G. O'Connel (WAM T105925); 1 male, same data (WAM T105944); 1 male, same data (WAM T105959); 1 male, Area C, 89.6 km NW. of Newman, 2254'31'S, 11900'26”E, 1 May 2010, wet pit trap, S. Ford, G. O'Connel (WAM T105908); 1 male, same data (WAM T105976); 1 female, Area C, 89.6 km NW. of Newman, 2300'03”S, 118 56'49”E, 24 February 2001, dug from burrow, Rocky gully, Z. Hamilton (WAM T101160); 1 male, Area C, 90.6 km NW. of Newman, 22 53'56”S, $119^{\circ} 00^{\prime} 07$ 'E, 1 May 2010, wet pit trap, S. Ford, G. O'Connel (WAM T105934); 1 male, Area C, $90.6 \mathrm{~km} \mathrm{NW.} \mathrm{of}$ Newman, 22 ${ }^{\circ} 59^{\prime} 52^{\prime \prime}$ S, 119 01'47’'E, 1 May 2010, wet pit trap, S. Ford, G. O'Connel (WAM T105915); 1 male, Area C, $92.7 \mathrm{~km} \mathrm{NW}$. of Newman, $23^{\circ} 01^{\prime} 04^{\prime \prime}$, 118 $54^{\circ} 24^{\prime \prime} \mathrm{E}, 24$ February 2010, dug from burrow, open mulga woodland with scattered eucalypts, M. Greenham (WAM T101153); 1 female, Area C, $98.2 \mathrm{~km} \mathrm{NW}$. of Newman, $23^{\circ} 00^{\prime} 28^{\prime \prime}$ S, 118 51'12'E, 21 February 2010, dug from burrow, open mulga woodland on clayey loam plain, M. Greenham (WAM T101166); 1 male, 36 km NW. of Balfour Downs Homestead, Pilbara Biological Survey site BDRN11, 22 30'59'S, 120³9'34'E, 11 September 2005-14 August 2006, wet pitfall, CALM Pilbara Survey

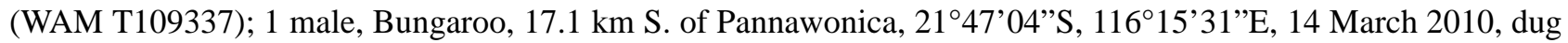
from burrow, J. Alexander (WAM T102531); 1 male, Bungaroo, same data except dry pit trap, J. Alexander (WAM T102533); 1 juvenile, same data except dug from burrow, D. Keirle (WAM T102536); 1 male, Bungaroo, $19.5 \mathrm{~km}$

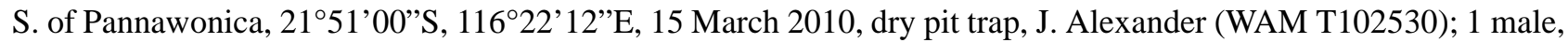
same data except 13 March 2010, D. Keirle (WAM T102532); 1 male, same data (WAM T102535); 1 male, Bunga-

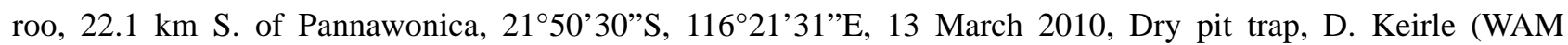

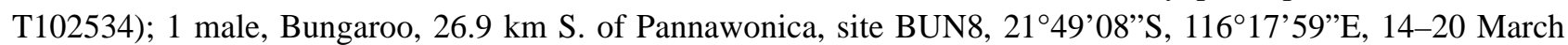
2005, dry pitfall traps, Z. Hamilton (WAM T72405); 1 male, Charles Darwin Reserve, $2.5 \mathrm{~km} \mathrm{~N}$. of White Wells Homestead, 29³3'29'S, 116 57'52'E, 4-5 March 2008, gas lantern/pitfall, mixed shrubland with emergent eucalypts and native pine, T.F. Houston (WAM T88785); 2 males, 9 km NE. of Cowra Line Camp, Pilbara Biological Survey site RHNW11, 22 17'38.8”S, 11903'40.9'”, 27 August 2003-20 October 2004, wet pitfall, CALM Pilbara Survey (WAM T109348); 1 male, 16 km N. of Cowra Line Camp, Pilbara Biological Survey site RHNW07, 22 $2^{\circ} 3^{\prime} 18.2^{\prime \prime}$ S, $119^{\circ} 01^{\prime} 29.5$ 'E, 14 August 2003-20 October 2004, wet pitfall, CALM Pilbara Survey (WAM T109353); 1 male, Davidson Creek, 75 km E. of Newman, vert site 1, 2331'29.81'’S, 120³8'28.26”'E, 8 April 2010, dry pitfall, J. Clark (WAM T102154); 1 male, Davidson Creek, $\sim 75 \mathrm{~km}$ E. of Newman, vert site 3,

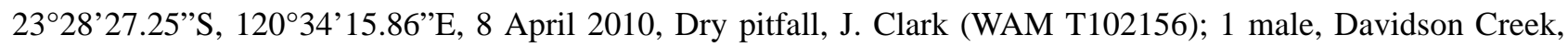

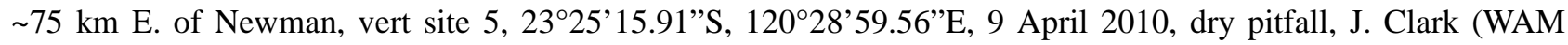
T102155); 1 male, Fortescue Marsh, 22 ${ }^{\circ} 17^{\prime} 32^{\prime \prime} S, 119^{\circ} 04^{\prime} 10^{\prime \prime} \mathrm{E}, 2-5$ June 2010, dry pitfall, leaf litter, P. Roberts, L. Quinn (WAM T107179); 1 male, same data (WAM T107180); 1 male, Fortescue Marsh, 22 ${ }^{\circ} 18$ '26”S, 
119 $12^{\prime}$ '57’E, 2-5 June 2010, dry pitfall, leaf litter, P. Roberts, L. Quinn (WAM T107182); 1 male, $67.5 \mathrm{~km}$ S. of Fortescue River crossing on Dampier Paraburdoo Railway, Pilbara Biological Survey site PE12, 22 03 '33.2”S, $117^{\circ} 41^{\prime} 36.5^{\prime}$ 'E, 5 September 2005-11 August 2006, wet pitfall, CALM Pilbara Survey (WAM T106274); 1 male, 7.4 km SW. of Fortescue Roadhouse, $21^{\circ} 20^{\prime} 58^{\prime \prime}$ S, 116 05'46”E, 24 March 2004, dug from burrow, M. Greenham,

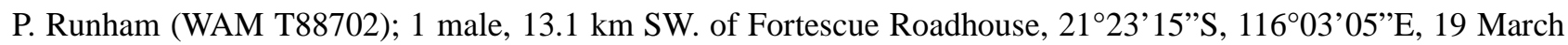
2004, dug from burrow, M. Greenham, P. Runham (WAM T88698); 1 male, same data except 20 March 2004 (WAM T88700); 1 male, same data except 22 March 2004 (WAM T88705); 1 male, same data (WAM T88708); 1

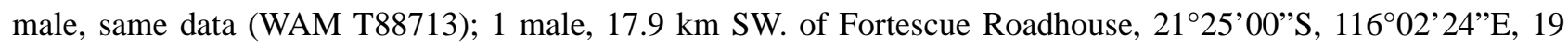
March 2004, dug from burrow, M. Greenham, P. Runham (WAM T88703); 1 male, same data (WAM T88706); 1 male, same data except 24 March 2004 (WAM T88707); 1 male, $18.4 \mathrm{~km} \mathrm{NE}$. of Fortescue Roadhouse,

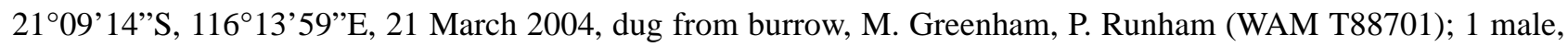
same data (WAM T88710); 1 male, same data (WAM T88712); 1 male, same data except 22 March 2004 (WAM

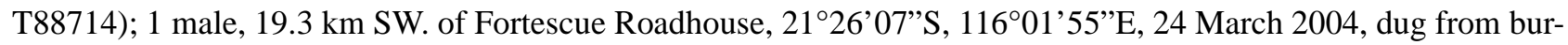
row, M. Greenham, P. Runham (WAM T88699); 1 male, $42 \mathrm{~km} \mathrm{~N}$. of Fortescue Roadhouse, 20 54'53”S, 116 ${ }^{\circ} 4^{\prime}$ 03”'E, 20 March 2004, dug from burrow, M. Greenham, P. Runham (WAM T88697); 1 male, same data (WAM T88709); 1 male, same data (WAM T88711); 2 males, Jimblebar minesite, $35 \mathrm{~km}$ E. of Newman, $23^{\circ} 22^{\prime} 09^{\prime}$ 'S, $120^{\circ} 11^{\prime} 44^{\prime \prime} \mathrm{E}, 9$ February 2009, pitfall trap, P. Bolton, C. Weston (WAM T95399 ${ }^{\mathrm{DNA}}$ ); 1 male, same data except $23^{\circ} 22^{\prime} 29^{\prime \prime}$ S, $120^{\circ} 12^{\prime} 37^{\prime \prime} \mathrm{E}, 12$ February 2009, active search (WAM T95402 ${ }^{\mathrm{DNA}}$ ); 1 male, same data except $23^{\circ} 22^{\prime} 45^{\prime}$ 'S, $120^{\circ} 06^{\prime} 59^{\prime \prime} \mathrm{E}, 9$ February 2009, pitfall trap (WAM T95400 ${ }^{\mathrm{DNA}}$ ); 1 male, same data except $23^{\circ} 23^{\prime} 27^{\prime \prime}$ 'S, $120^{\circ} 05^{\prime} 43^{\prime \prime} \mathrm{E}, 6$ February 2009, active search (WAM T95401 ${ }^{\text {DNA }}$ ); 1 male, Jinayri, $c a .65 \mathrm{~km} \mathrm{NW}$. of

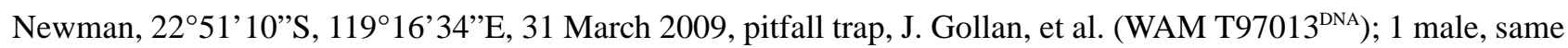
data except $22^{\circ} 54^{\prime} 37^{\prime}$ 'S, $119^{\circ} 12^{\prime} 30^{\prime \prime}$ E, 1 April 2009 (WAM T97033 $3^{\mathrm{DNA}}$ ); 7 males, $3.5 \mathrm{~km}$ S. of Karratha, Pilbara Biological Survey, site DRC01, 2046'4.7'S, 116 50'31.3”E, 1 July 2003-2 October 2004, wet pitfall, CALM Pilbara Survey (WAM T109322); 1 male, 6 km S. of Karratha, Pilbara Biological Survey site DRC03, 2047'40.1"'S, 116 51'24.1'E, 3 July 2003-3 October 2004, wet pitfall, CALM Pilbara Survey (WAM T109354); 1 male, 5 km N. of Lake Poongkaliyarra, Pilbara Biological Survey site DRC07, 2056’04.4”S, 11706'54.3”'E, 4 July 2003-3 October 2004, wet pitfall, DEC Pilbara survey (WAM T109323); 1 male, 9 km NW. of Lake Poongkaliyarra, Pilbara Biological Survey site DRC05, 2056'23.8'S, $17^{\circ} 02^{\prime} 05.3^{\prime \prime}$ E, 3 July 2003, 3 October 2004, wet pitfall,

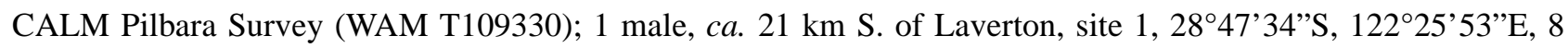
January 2011, pit trap, S.A. Thompson (WAM T110258); 1 male, same data (WAM T110263); 1 male, same data (WAM T110269); 1 male, same data (WAM T110272); 1 male, ca. $21 \mathrm{~km} \mathrm{~S}$. of Laverton, site 1, 31 ${ }^{\circ} 20^{\prime} 52^{\prime \prime}$, $119^{\circ} 23^{\prime} 26^{\prime}$ E, 6 January 2011, pit trap, S.A. Thompson (WAM T110264); 1 female, ca. $21 \mathrm{~km} \mathrm{S.} \mathrm{of} \mathrm{Laverton,} \mathrm{site}$ 11, 28 49'35'S, 122 23'20”'E, 8 January 2011, pit trap, S.A. Thompson (WAM T110273); 1 male, ca. $21 \mathrm{~km} \mathrm{S.} \mathrm{of}$ Laverton, site 13, 28 $8^{\circ} 9^{\prime} 11^{\prime \prime} S, 122^{\circ} 23^{\prime} 14^{\prime \prime}$ E, 6 January 2011, pit trap, S.A. Thompson (WAM T110276); 1 male, ca. $21 \mathrm{~km} \mathrm{~S}$. of Laverton, site 2, $28^{\circ} 47^{\prime} 55^{\prime \prime} \mathrm{S}, 1^{\circ} 2^{\circ} 26^{\prime} 01^{\prime \prime} \mathrm{E}, 6$ January 2011, pit trap, S.A. Thompson (WAM T110265); 1 male, same data except 8 January 2011 (WAM T110270); 1 male, $c a .21 \mathrm{~km} \mathrm{~S}$. of Laverton, site 5, $28^{\circ} 49^{\prime} 07^{\prime \prime}$ S, $122^{\circ} 26^{\prime} 02^{\prime \prime}$ E, 6 January 2011, pit trap, S.A. Thompson (WAM T110271); 1 male, ca. $21 \mathrm{~km} \mathrm{S.} \mathrm{of}$

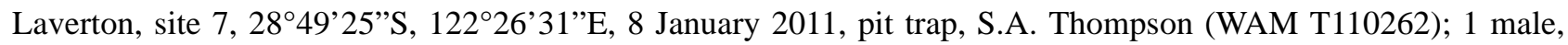

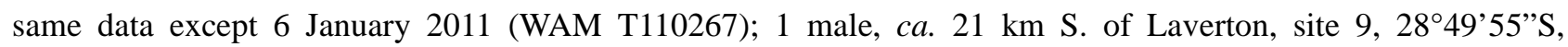
$122^{\circ} 25^{\prime} 34^{\prime \prime}$, 8 January 2011, pit trap, S.A. Thompson (WAM T110257); 1 male, Lorna Glen Biological Survey,

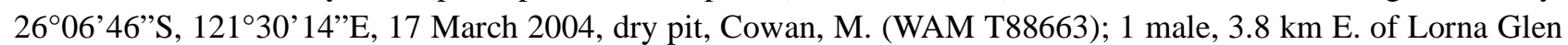

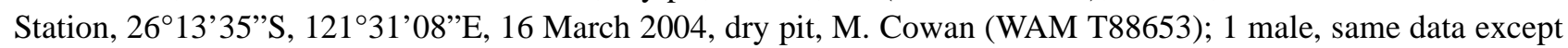

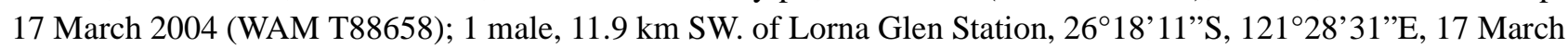
2004, dry pit, M. Cowan (WAM T88659); 1 male, $25.2 \mathrm{~km} \mathrm{~N}$. of Lorna Glen Station, 2600'05”S, 121³3'48”'E, 16 March 2004, dry pit, M. Cowan (WAM T88656); 1 male, Mallina Station, SW. of homestead, Pilbara Biological Survey site DRE12, 2054'36”S, $117^{\circ} 58^{\prime} 58^{\prime}$ 'E, 14 November 2003-12 May 2004, wet pitfall, CALM Pilbara Survey (WAM T109343); 5 males, $10 \mathrm{~km} \mathrm{S.} \mathrm{of} \mathrm{Mallina} \mathrm{Homestead,} \mathrm{Pilbara} \mathrm{Biological} \mathrm{Survey} \mathrm{site} \mathrm{DRE13,}$ 20 58'10.4”'S, $118^{\circ} 02^{\prime} 54.0^{\prime \prime}$ E, 10 July 2003-3 October 2004, wet pitfall, CALM Pilbara Survey (WAM T109327);

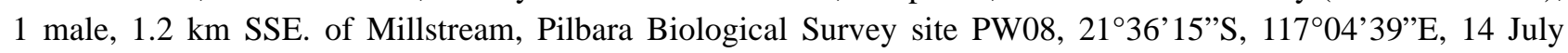
2003-12 October 2004, wet pitfall, CALM Pilbara Survey (WAM T109336); 1 juvenile, Mesa A, $43.9 \mathrm{~km} \mathrm{~W}$. of

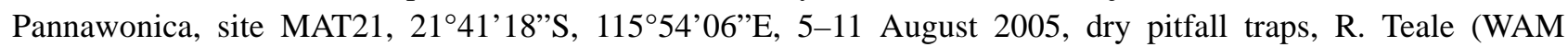
T72402); 1 male, Mesa J, 16.6 km SW. of Pannawonica, site MEJ1, 2145'34”S, 116¹3’39”E, 14-20 March 2005, 
dry pitfall traps, D. Kamien (WAM T72400); 2 males, same data (WAM T72401); 3 males, Mesa J, 17 km SW. of

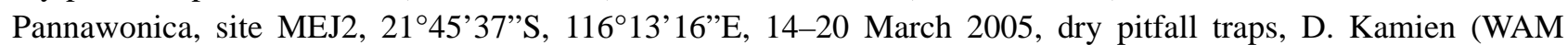
T72399); 3 males, $2.5 \mathrm{~km} \mathrm{~N}$. of Mile Camp, Pilbara Biological Survey site RHNE02, 22 $2^{\circ} 5^{\prime} 57.8^{\prime \prime}$, 119³7'53.1'E, 10 August 2003-19 October 2004, wet pitfall, CALM Pilbara Survey (WAM T109321); 1 male,

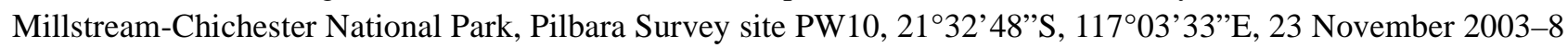
May 2004, wet pitfall trap, CALM Pilbara Survey (WAM T109320); 1 male, 24 km ENE. of Moorimoordinina Native Well, Pilbara Biological Survey site RHNE08, 22²7'07'S, 11958'34'E, 9 August 2003-20 October 2004, wet pitfall, CALM Pilbara Survey (WAM T109335); 4 males, $10.5 \mathrm{~km} \mathrm{NW.} \mathrm{of} \mathrm{Mt} \mathrm{Berry,} \mathrm{Pilbara} \mathrm{Biological} \mathrm{Survey}$ site WYE12, 22²4'38.6”'S, 116 23'30.8'E, 10 September 2003-9 October 2004, wet pitfall, CALM Pilbara Survey (WAM T109341); 2 males, 19.7 km WNW. of Mt Berry, Pilbara Biological Survey site WYE09, 22²6'15.3”'S, $116^{\circ} 16^{\prime} 27.3$ ”'E, 8 September 2003-10 October 2004, wet pitfall, CALM Pilbara Survey (WAM T109344); 2 males, 8 km NNE. of Mt Edith, Pilbara Biological Survey, site WYE05, 22³4'16.9”'S, 116 08'53.8'E, 11 September 2003-7 October 2004, Ethylene Glycol Pit, CALM Pilbara Survey (WAM T109345); 1 male, 14.5 km SE. of Mt Florance Homestead, Pilbara Biological Survey site PE06, 2151'22.2”'S, 11758'47.3”E, 3 September 2003-11 October 2004, wet pitfall, CALM Pilbara Survey (WAM T109362); 6 males, 24 km WSW. of Mt Marsh, Pilbara Biological Survey site RHNW02, 22³2'09”S, 11859'51'E, 12 August 2003-19 October 2004, wet pitfall, CALM Pilbara Survey (WAM T109329); 1 male, 26 km WSW. of Mt Marsh, Pilbara Biological Survey site RHNW01, 22 32'06'S, 118 58'39’E, 12 August 2003-19 October 2004, wet pitfall, CALM Pilbara Survey (WAM T109350); 1 male, 7 km SSE. of Mt Minnie, Pilbara Biological Survey site WYW03, 22 ${ }^{\circ} 10^{\prime} 10.8^{\prime \prime S}$, 115'33'39”'E, 27 September 2003-30 September 2004, wet pitfall, CALM Pilbara Survey (WAM T109325); 1 male, Mount Webber, 20³1'21'S, 119 ${ }^{\circ} 18^{\prime} 41^{\prime \prime}$ 'E, 31 March 2010-7 May 2010, wet pitfall trap, gorge, P. Bolton

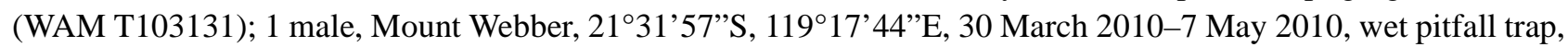

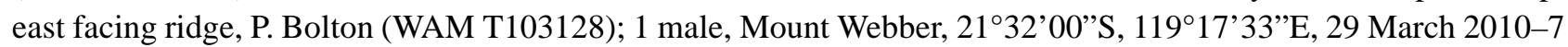
May 2010, wet pitfall trap, gorge, P. Bolton (WAM T103129); 2 males, Mt Stuart Station, NE. of homestead, Pilbara Survey site WYE13, $22^{\circ} 25^{\prime} 30^{\prime \prime}$ S, $116^{\circ} 25^{\prime} 57^{\prime \prime}$, 1 May 2004-10 October 2004, wet pitfall, CALM Pilbara

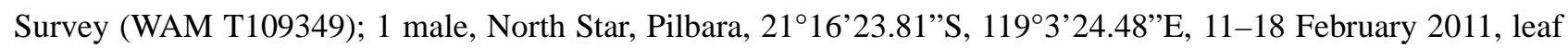
litter, Thompson (WAM T112591); 1 male, $12.5 \mathrm{~km} \mathrm{~N}$. of Nullagine, Pilbara Biological Survey site NW03, $21^{\circ} 46^{\prime} 13.1^{\prime \prime}$ S, $120^{\circ} 05^{\prime} 30.7$ 'E, 5 August 2003-18 October 2004, wet pitfall, CALM Pilbara Survey (WAM

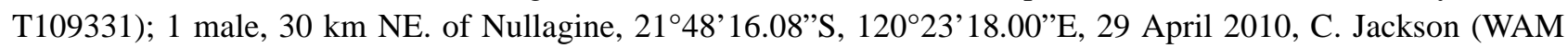
T102065); 2 males, $56.3 \mathrm{~km} \mathrm{~N}$. of Nullagine, Pilbara Biological Survey site NW04, 21 ${ }^{\circ} 40^{\prime} 42.7^{\prime \prime} \mathrm{S}, 120^{\circ} 05^{\prime} 18.2^{\prime \prime} \mathrm{E}$, 19 May 2004-18 May 2005, ethylene glycol pits, CALM Pilbara Survey (WAM T104454); 1 male, Orebody 35, ca. $8 \mathrm{~km}$ W. of Newman, Site10-P6, 2323'59.65'S, 119³9'07.47’'E, 12 March 2010-18 May 2010, pitfall trap, open floodplain, J. Gollan (WAM T104794); 1 male, Orebody 35, ca. $8 \mathrm{~km} \mathrm{W.} \mathrm{of} \mathrm{Newman,} \mathrm{Site3-HC1,}$ $23^{\circ} 24^{\prime} 17.00^{\prime \prime} \mathrm{S}, 19^{\circ} 36^{\prime} 18.90^{\prime} \mathrm{E}, 15$ March 2010 , hand collected, drainage gully, south facing ridge, deeply vegetated, J. Gollan, M. Bulbert, M. Semeniuk, J. Bevan (WAM T104784); 1 male, Orebody 35, ca. 8 km W. of New-

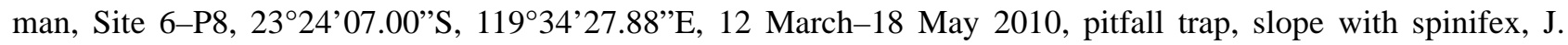
Gollan (WAM T104810); 1 male, Orebody 35, ca. 8 km W. of Newman, Site9-P10, 2323'22.32”S, 119³8'36.06”'E, 12 March-18 May 2010, pitfall trap, open floodplain, J. Gollan (WAM T104807); 1 male, 2.3 km

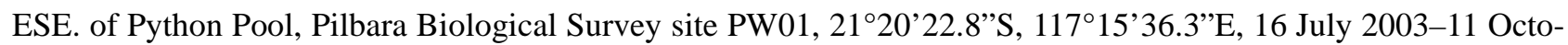
ber 2004, wet pitfall, CALM Pilbara Survey (WAM T109356); 2 males, 5 km WSW. of Python Pool, Pilbara Bio-

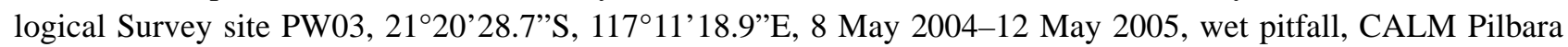
Survey (WAM T109339); 5 males, 1 juvenile, $11.5 \mathrm{~km} \mathrm{SW}$. of Rhodes Ridge, Pilbara Biological Survey site RHNC07, $23^{\circ} 08^{\prime} 45.7^{\prime}$ 'S, $119^{\circ} 15^{\prime} 56.9^{\prime \prime}$ E, 25 May 2004-11 May 2005, wet pitfall, CALM Pilbara Survey (WAM T109324); 1 male, Roy Hill, 22²5'35.77'S, 11956'32.69'E, 11-17 March 2010, wet pitfall trap, N. Thompson, L. Roque-Albelo (WAM T102618); 1 female, South Parmelia, 66 km NW. of Newman, 2302'08'S, 119 11'17'E, 12 April 2011, dug from burrow, R. Teale, M. Greenham (WAM T113574); 1 female, same data except

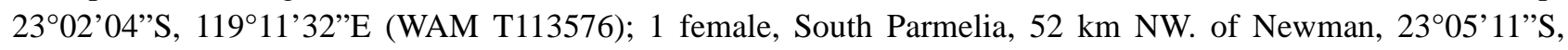
119¹9'07'E, 16 April 2011, dug from burrow, R. Teale, M. Greenham (WAM T113582); 1 female, Southern Flank, $71 \mathrm{~km}$ NW. of Newman 22 $59^{\prime} 40^{\prime}$ 'S, $119^{\circ} 09^{\prime} 55^{\prime}$ E, 17 April 2011, dug from burrow, R. Teale, M. Greenham (WAM T113612); 1 male, Tom Price, $22^{\circ} 42^{\prime} \mathrm{S}, 17^{\circ} 47^{\prime} \mathrm{E}, 3$ January 1994, M. Hyams (WAM T30075, formerly 94/

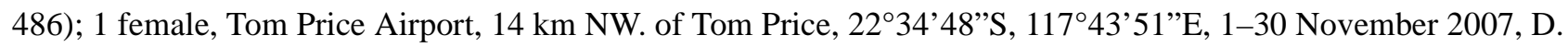
Kamien, J. Adcroft (WAM T102911); 2 males, Warramboo, $50.5 \mathrm{~km} \mathrm{~W}$. of Pannawonica, site WA3, 2140'00”S, 
115 50'06”'E, 14-20 March 2005, dry pitfall traps, D. Kamien (WAM T72404); 1 male, Warramboo, $52.1 \mathrm{~km} \mathrm{W.} \mathrm{of}$

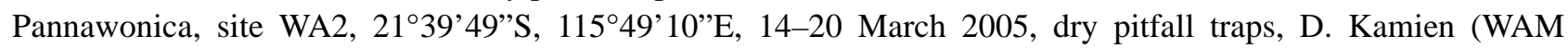

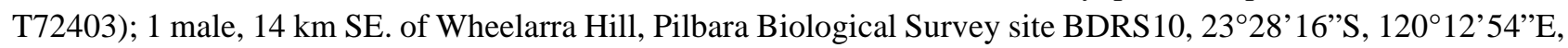
6 September 2005-10 August 2006, wet pitfall, CALM Pilbara Survey (WAM T109352); 1 male, 34 km E. of Wheelarra Hill, Pilbara Biological Survey site BDRS03, 2321'31'S, 120²7’33”E, 4 September 2005-11 August 2006, wet pitfall, CALM Pilbara Survey (WAM T109347); 1 male, $11 \mathrm{~km}$ SSE. of Whim Creek Hotel, Pilbara Biological Survey site DRE10, 2055'11.4’S, 11751'40.6”E, 11 July 2003-3 October 2004, ethylene glycol pits, CALM Pilbara Survey (WAM T109333); 2 males, 1 female, 2 juveniles, $5 \mathrm{~km}$ E. of Whim Creek Hotel, Pilbara Biological Survey site DRE08, 2050'54.6”S, 11751'16.2”E, 11 July 2003-3 October 2004, ethylene glycol pits, CALM Pilbara Survey (WAM T109319); 1 male, ca. $7 \mathrm{~km}$ NE. of Wodgina mine site, $21^{\circ} 09^{\prime} 10.04^{\prime \prime}$, 118 43'10.03'"E, 1 April-4 May 2010, wet pitfall trap, minor drainage line, P. Bolton (WAM T107002); 1 male, $c a$.

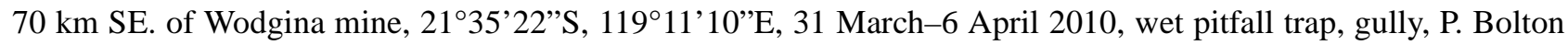
(WAM T105227); 1 male, same data (WAM T105228); 1 male, 8.5 km WSW. of Yanyare River Mouth, Pilbara Biological Survey site DRW02, 2050'40.6”S, 116 22'02.9'E, 23 September 2003-2 October 2004, wet pitfall, CALM Pilbara Survey (WAM T109326).

Etymology. The specific epithet refers to the yellow-brown colouration of parts of the carapace (mellosa, Latin, of honey, honey-coloured) (Brown 1956).

Diagnosis. Males of A. mellosa differ from all other named species of the genus by the colouration of the carapace which is overall light yellowish-brown with the cephalic region honey-coloured to very dark reddish-brown (Fig. 10). Females also have a somewhat darker cephalic region but the colour difference between the cephalic and thoracic regions is much less pronounced (Fig. 21). Tibia I of males has a large spur and megaspine located medially, and metatarsus I is incrassate with the 'heel' of the thickening about half-way; this conformation most closely resembles A. pallida from eastern Queensland from which it differs by the less pronounced metatarsal process and the smaller tibial spur and megaspine. Female genitalia resemble those of A. longitheca Raven, 1984 from central Queensland in possessing long lateral tubes (Figs 23, 24), but they differ in the shape of the median spermathecae which are coiled in A. longitheca and S-shaped in A. mellosa. See Remarks for further clarification.

Description. Adult male (based on holotype WAM T94870): medium-sized nemesiid spider (total length 18.4).

Colour: carapace (Fig. 10) light yellow-brown in thoracic region turning darker towards anterior parts, cephalic region dark reddish-brown, eye region black (Fig. 13); sternum light yellow-brown, sigilla and labium largely brown (Fig. 12); abdomen dorsally dark brownish-grey, mottled with small light-brown spots (Fig. 11), ventrally light yellowish; chelicerae very dark reddish-brown; pedipalp segments reddish-brown, tibia somewhat lighter (Figs 17, 18); legs light yellowish-brown (Figs 15, 16).

Carapace: 7.0 long, 5.6 wide; eye group (Fig. 13) 1.4 wide, 0.7 long; fovea procurved (Fig. 10).

Sternum and labium: 4.4 long, 2.8 wide; 2 pairs of elongated sigilla in posterior half (Fig. 12); labium wider than long, slightly indented anteriorly (Fig. 14).

Maxillae: with > 50 pin-like cuspules, extending to heel (Fig. 14).

Chelicerae: without rastellum (Fig. 12), promargin with 9 large teeth, retromargin with 4 smaller teeth proximally.

Abdomen: 7.4 long, 5.0 wide (Fig. 11). Four spinnerets, terminal segment of posterior lateral spinnerets digitiform.

Pedipalp: length of femur 4.0, patella 2.2, tibia 3.1, tarsus 3.1. Femur with 1 disto-prolateral spine; patella with 3 prolateral spines; tibia with 2 baso-ventral spines and 7-8 prolateral spines in apical half, tibia with slight distoventral depression without setae (accommodates bulb and embolus) (Figs 17, 18); tarsus terminally blunt; bulb globular, embolus ca. 0.8 long, slightly curved and tapering (Figs 17-20).

Legs: femur I with row of 7 dorsal spines in basal half and 2 disto-prolateral spines, patella with 2 prolateral spines, metatarsi and tarsi without spines. Tibia I with large spur and megaspine, metatarsi incrassate (Fig. 16). Tarsi and apical half of metatarsi of legs I and II ventrally with entire dense scopulae, tarsi of legs III and IV with divided weak scopulae. Tarsi, metatarsi and tibiae with numerous dorsal trichobothria of variable length. Paired tarsal claws of legs with two rows of 7-10 teeth each; third tarsal claw reduced and very small. 

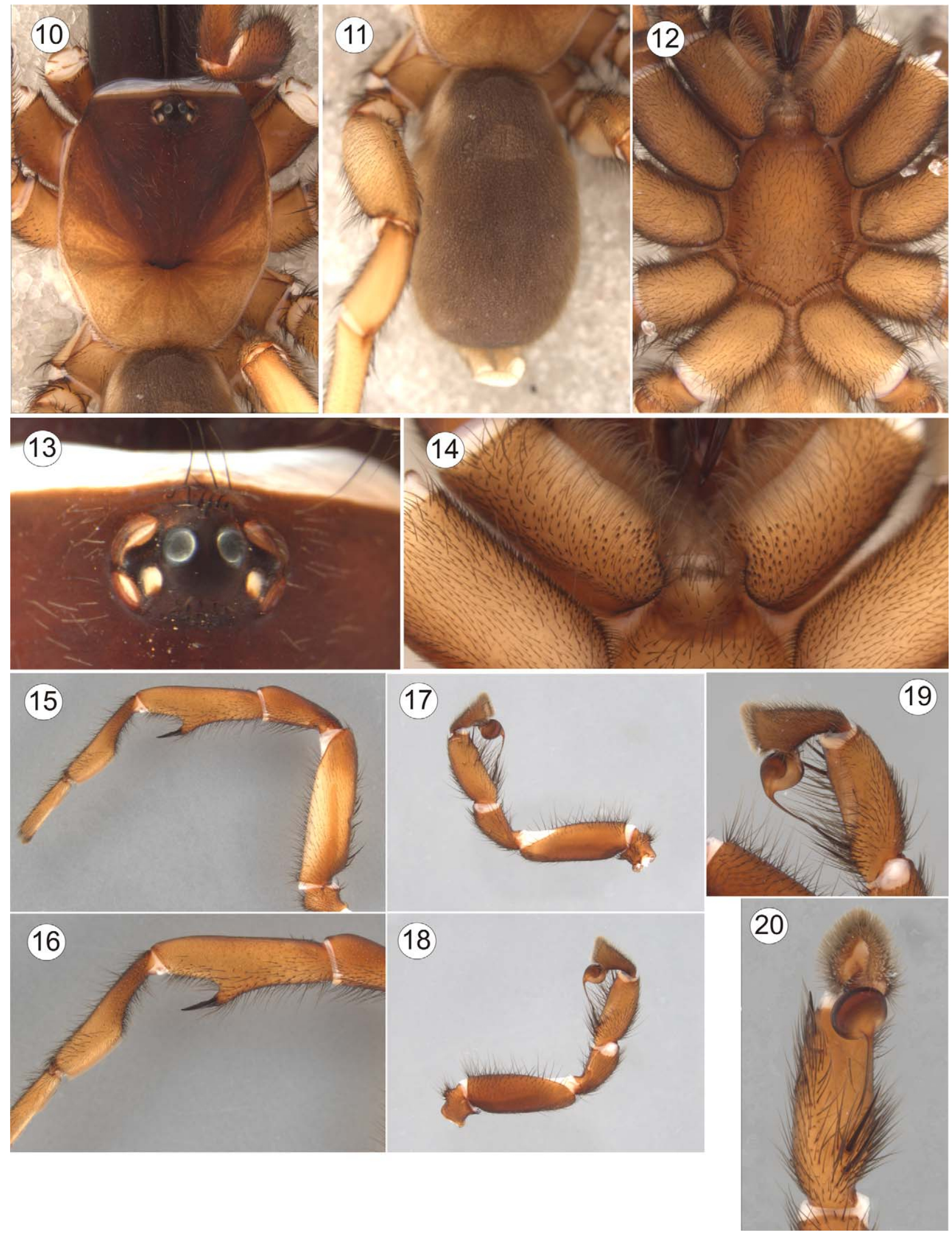

FIGURES 10-20. Aname mellosa $\mathbf{n}$. sp., holotype male (WAM T94870): 10, carapace, dorsal view; 11, abdomen, dorsal view; 12, cephalothorax, ventral view; 13, eyegroup, dorsal view; 14, maxillae, labium and anterior region of sternum, ventral view; 15, left leg I, retrolateral view; 16, left tibia and metatarsus I, retrolateral view; 17, left pedipalp, prolateral view; 18, left pedipalp, retrolateral view; 19, left pedipalpal tibia and tarsus, retrolateral view; 20, same, ventral view. 
Leg measurements: length of legs I $>\mathrm{IV}>\mathrm{II}>\mathrm{III}$. leg I: femur 6.0, patella 3.5, tibia 4.6, metatarsus 4.2, tarsus 2.5 , total $=20.8$. Dorsal proximal width of patella $\mathrm{I}=1.4$, tibial index $=0.12$, leg I formula $=2.97$. Leg II: femur 6.0, patella 3.4, tibia 4.5, metatarsus 4.9, tarsus 3.1, total $=21.9$. Dorsal proximal width of patella $\mathrm{II}=1.2$, tibial index $=$ 0.15; leg II formula $=3.13$. Leg III: femur 5.2, patella 3.0, tibia 3.2, metatarsus 6.6, tarsus 2.9, total = 20.9. Dorsal proximal width of patella III $=1.3$, tibial index $=0.19$; leg III formula $=2.99$. Leg IV: femur 7.0, patella 3.5, tibia 5.9 , metatarsus 6.3, tarsus 2.9, total $=25.6$. Dorsal proximal width of patella IV $=1.3$, tibial index $=0.14$; leg III formula $=3.66$.

Variation: total length 5.1-6.2, carapace 6.1-7.9 long, 16.9-20 wide ( $n=12)$.

Adult female (based on WAM T96150): medium-sized nemesiid spider (total length 19.5).

Colour: light yellowish-brown with indistinctly darker cephalic region (Fig. 21), eye region brown; sternum, sigilla and labium uniformly light yellowish-brown; abdomen dorsally dark greyish-brown with purple tinge and mottled with small lighter small spots, ventrally light yellowish-brown; chelicerae orange-brown; pedipalps and legs uniformly light yellow-brown.

Carapace: 7.5 long, 6.4 wide; eye group 1.4 wide, 0.8 long; fovea slightly procurved.

Sternum and labium: 5.0 long, 3.2 wide (Fig. 22); 2 pairs of indistinct elongated sigilla in posterior half, 1 pair of round sigilla at base of leg II; labium wider than long, slightly indented anteriorly.

Maxillae: with > 100 pin-like cuspules, extending to heel and covering in low density almost entire retrolateral half of maxillae.

Chelicerae: without rastellum, promargin with 9 large teeth, retromargin with 4 smaller teeth proximally.

Abdomen: 8.9 long, 5.9 wide. Four spinnerets, terminal segment of posterior lateral spinnerets digitiform.

Genitalia: median spermathecal stalks S-shaped, spermathecae slightly wider than spermathecal stalks; separate straight and elongated tube accompanies spermatheca (Figs 23, 24).

Pedipalp: length of femur 3.6, patella 2.3, tibia 2.5, tarsus 2.7. Femur without spine, patella with 2 prolateral spines; tibia with 11-13 irregularly placed spines in mainly apical half (not dorsal); tarsus with scopulae ventrally and 2 baso-ventral spines and curved terminal claw.

Legs: femur I with 1 dorsal spines proximally and 1 disto-prolateral spine, patella with 2 (left leg 1) prolateral spine, tibia with a row of three ventral spines ( 5 on right leg of which 2 are paired) and 2 disto-ventral spines, metatarsi 1 basal and 1 apical pair of ventral spines (left leg) ( 3 additional medio-ventral spines on left leg). Tarsi and apical half of metatarsi of leg I and II ventrally with entire dense scopulae, tarsi of legs III and IV with divided very weak scopulae. Tarsi, metatarsi and tibiae with numerous dorsal trichobothria of variable length. Paired tarsal claws of legs with two rows of 10-13 (legs I-II) or 5-7 (legs III-IV) teeth each; third tarsal claw reduced and very small.

Leg measurements: length of legs IV $>\mathrm{I}>\mathrm{II}>\mathrm{III}$. leg I: femur 6.0, patella 3.1, tibia 4.0, metatarsus 3.5, tarsus 2.3, total $=18.9$. Dorsal proximal width of patella $\mathrm{I}=1.0$, tibial index $=0.14$, leg $\mathrm{I}$ formula $=2.12$. Leg II: femur 5.0, patella 3.0, tibia 3.3, metatarsus 3.1, tarsus 2.1, total $=16.5$. Dorsal proximal width of patella $\mathrm{II}=0.9$, tibial index $=$ 0.14; leg II formula $=1.85$. Leg III: femur 4.0, patella 2.5, tibia 2.0, metatarsus 2.9, tarsus 1.7, total = 13.1. Dorsal proximal width of patella III $=1.1$, tibial index $=0.24$; leg III formula $=1.47$. Leg IV: femur 5.5 , patella 3.2 , tibia 4.0, metatarsus 4.4, tarsus 2.2 , total $=19.3$. Dorsal proximal width of patella IV $=1.1$, tibial index $=0.15$; leg III formula $=2.17$.

Variation: total length 19.3-25.0, carapace 5.4-6.6 long, 6.6-8.7 wide $(n=8)$.

Distribution. Aname mellosa has been found throughout the central Pilbara, from Jimblebar minesite near the township of Newman in the east to Mount Minnie in the west, and south into the Midwest region of Western Australia (Fig. 5).

Remarks. Care must be taken when identifying A. mellosa as a number of undescribed species from northern Western Australia have a very similar somatic appearance, but have a different genital morphology. The embolus of male A. mellosa is long and slightly curved, but it is often much shorter and/or much more curved in the undescribed species. An unambiguous identification of females or juveniles in this group is currently not possible, however, we have listed females and juveniles when they have been collected with or in close proximity to males. As stated in the Diagnosis, females of A. mellosa have an unusual genital morphology with long, straight lateral tubes in addition to the median pair of spermathecae. This morphology is similar to that of A. longitheca (Raven 1985a), but both species differ in the shape of the median spermathecae. 


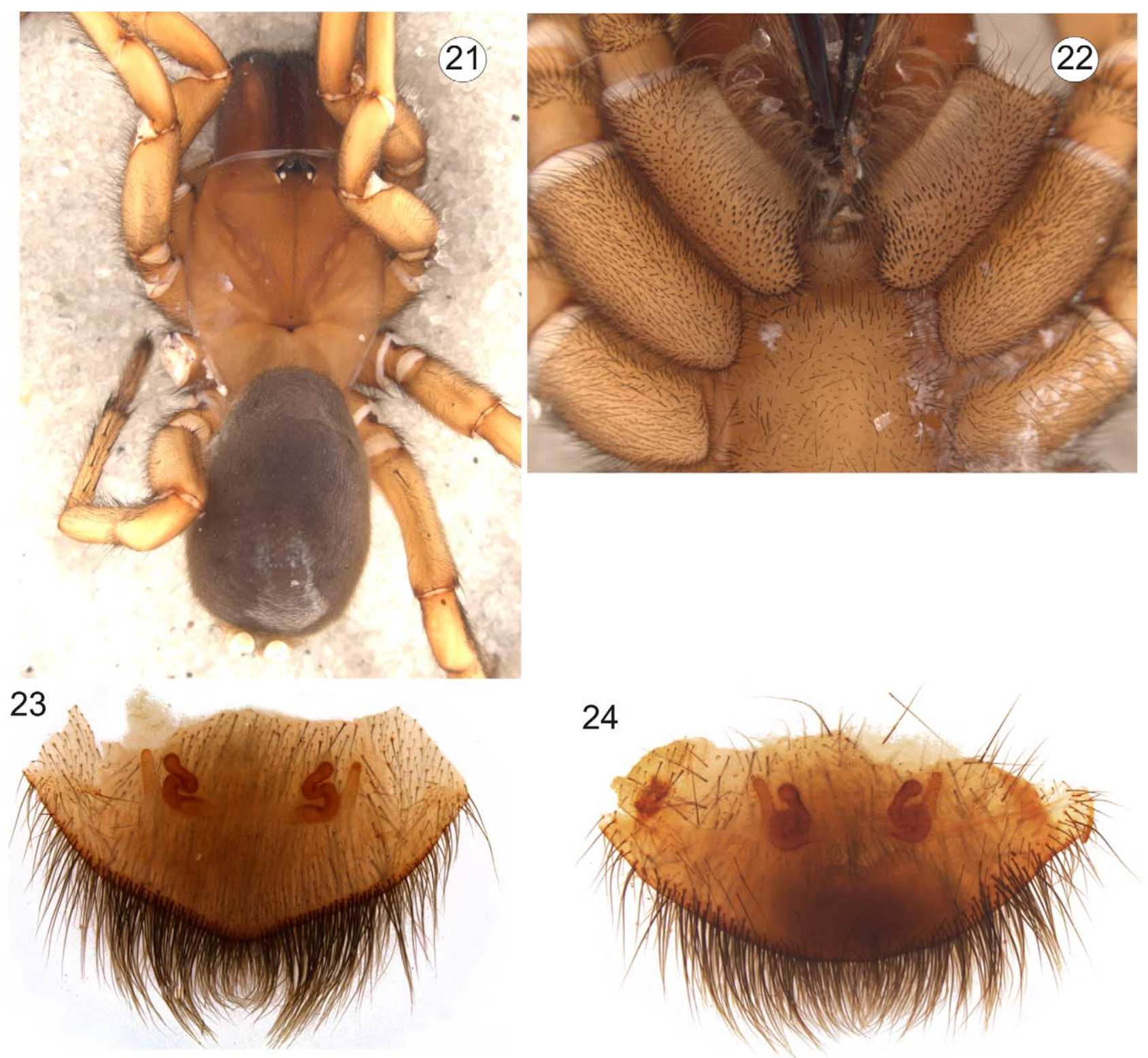

FIGURES 21-24. Aname mellosa n. sp., females: 21, dorsal view (WAM T96145); 22, maxillae, labium and anterior region of sternum, ventral view (WAM T96145); 23, genitalia, dorsal view (WAM T101163); 24, genitalia, dorsal view (WAM T102911).

All records of mature males were collected between January and early June suggesting this species to be reproductively active towards the end of the wet season. Many mature males of A. mellosa were removed from their burrows (R. Teale, pers. comm.) where it is likely that they had matured and were waiting for suitable conditions to emerge and search for adult females. The silk-lined open burrow of A. mellosa is dug at an angle into the substrate and lacks a lid (Figs 2-4) (R. Teale, pers. comm.).

\section{Aname aragog n. sp.}

Figs 7, 9, 25-33

Type material. AUSTRALIA: Western Australia: holotype male, Jimblebar minesite, $35 \mathrm{~km}$ E. of Newman, $23^{\circ} 22^{\prime} 44^{\prime \prime}$, $120^{\circ} 15^{\prime} 27^{\prime \prime}$, 8 February 2009, P. Bolton, C. Weston (WAM T95404 ${ }^{\text {DNA }}$ ).

Etymology. This species is named for J. K. Rowling's fictional spider Aragog which first appeared in Harry Potter and the Chamber of Secrets (Rowling 1998). The name is to be treated as a noun in apposition. 

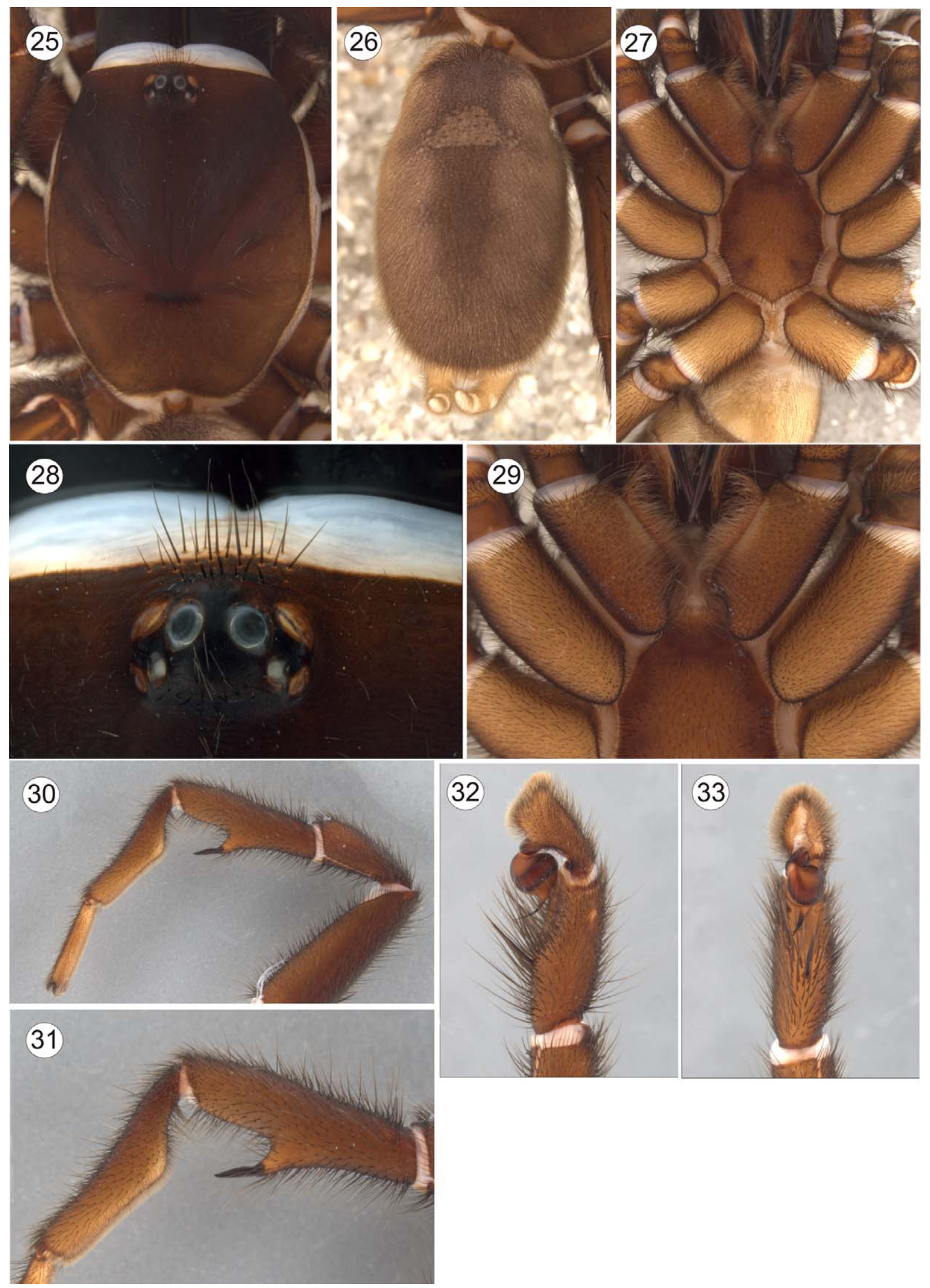

FIGURES 25-33. Aname aragog n. sp., holotype male (WAM T95404): 25, carapace, dorsal view; 26, abdomen, dorsal view; 27, cephalothorax, ventral view; 28, eye group; 29, maxillae, labium and anterior region of sternum, ventral view; 30, left leg I, retrolateral view; 31, left tibia and metatarsus I, retrolateral view; 32, left pedipalpal tibia and tarsus, retrolateral view; 33, same, ventral view. 
Diagnosis. Males of A. aragog are unique in the genus in having metatarsus I with a short and shallow basal excavation, and a very shallow proximal edge of the process on tibia I (Fig. 31). Males of A. aragog differ from other Pilbara species described here by their overall darker colouration (Figs 25-31) and larger size. The embolus is much shorter than that of A. mellosa (Fig. 32), and the pedipalpal bulb is much more bulbous than in A. ellenae and A. marae (Fig. 32). Females of A. aragog are unknown.

Description. Adult male (based on holotype WAM T95404): medium-sized nemesiid spider (total length 27.2).

Colour: carapace brown with somewhat darker cephalic region (Fig. 25), eye region brown (Fig. 28); sternum light brown, darker towards margins, sigilla and labium brown (Fig. 27); abdomen dorsally dark greyish brown, centrally darker with dark purple tinge, ventrally light brown (Fig. 26); chelicerae glabrous very dark brown to black; pedipalp segments uniformly dark brown (Fig. 32), legs brown, metatarsi and tarsi somewhat lighter in particular ventrally (Fig. 30).

Carapace: 8.9 long, 6.7 wide; eye group (Fig. 28) 1.5 wide, 0.8 long; fovea slightly procurved (Fig. 25).

Sternum and labium: 5.2 long, 3.5 wide; 2 pairs of indistinct elongated sigilla in posterior half (Fig. 27); labium wider than long, slightly indented anteriorly (Fig. 29).

Maxillae: with > 150 pin-like cuspules, extending to heel and to centre of maxillae (Fig. 29).

Chelicerae: without rastellum, promargin with 9 large teeth, retromargin with 5 smaller teeth proximally.

Abdomen: 10.0 long, 5.9 wide (Fig. 26). Four spinnerets, terminal segment of posterior lateral spinnerets digitiform.

Pedipalp: length of femur 4.7, patella 2.8, tibia 3.1, tarsus 3.1. Femur and patella without spines; tibia with 1 medio-ventral spines and 1 ventro-prolateral spines in apical half, tibia with indistinct disto-ventral depression without setae (accommodates bulb and embolus) (Fig. 32); tarsus terminally blunt; bulb globular, embolus ca. 0.6 long, curved mainly at base and tapering (Figs 32, 33).

Legs: femur I with 1 dorsal spine in basal half and 1 disto-prolateral spine, patella with 2 prolateral spines, metatarsi and tarsi without spines. Tibia I with large spur and megaspine, metatarsi incrassate (Figs 30, 31). Tarsi and apical half of metatarsi of leg I and II ventrally with entire dense scopula, tarsi of legs III and IV with divided scopula. Tarsi, metatarsi and tibiae with numerous dorsal trichobothria of variable length. Paired tarsal claws of legs with two rows of 8-12 teeth each; third tarsal claw reduced and very small.

Leg measurements: length of legs IV >I $>$ II $>$ III. leg I: femur 6.5, patella 4.0, tibia 5.0, metatarsus 5.1, tarsus 3.1, total $=23.7$. Dorsal proximal width of patella $\mathrm{I}=1.8$, tibial index $=0.2$, leg $\mathrm{I}$ formula $=2.66$. Leg II: femur 6.0, patella 3.4, tibia 4.5, metatarsus 4.9, tarsus 3.1, total $=21.9$. Dorsal proximal width of patella $\mathrm{II}=1.5$, tibial index $=$ 0.19; leg II formula $=2.46$. Leg III: femur 5.2, patella 3.0, tibia 3.2, metatarsus 6.6, tarsus 2.9, total = 20.9. Dorsal proximal width of patella III $=1.5$, tibial index $=0.24$; leg III formula $=2.35$. Leg IV: femur 7.0, patella 3.5 , tibia 5.9 , metatarsus 6.3, tarsus 2.9, total $=25.6$. Dorsal proximal width of patella IV $=1.6$, tibial index $=0.17$; leg IV formula $=2.87$.

Variation: unknown.

Adult female: unknown.

Distribution. Aname aragog has been found at a single location at the Jimblebar minesite, $c a$. $35 \mathrm{~km} \mathrm{E}$. of the township of Newman (Fig. 6).

\section{Aname ellenae n. sp.}

Figs 6, 9, 34-42

Type material. AUSTRALIA: Western Australia: holotype male, Aquila Onslow, $24.9 \mathrm{~km}$ SE. of Onslow, $21^{\circ} 46^{\prime} 56^{\prime}$ 'S, $115^{\circ} 17^{\prime} 40^{\prime}$ E, 28 October 2008, dug from burrow, M. Menz (WAM T98890 ${ }^{\mathrm{DNA}}$ ). Paratypes: 2 males, same data as holotype except 29 October 2008 (WAM T98773 ${ }^{\text {DNA }}$, T98893 ${ }^{\mathrm{DNA}}$ ).

Other material examined. AUSTRALIA: Western Australia: 17 males, Aquila Onslow, $14.7 \mathrm{~km} \mathrm{S.} \mathrm{of}$

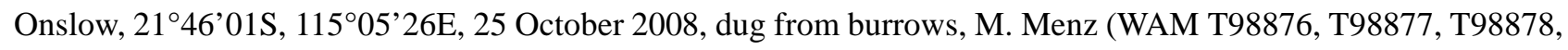
T98879, T98880, T98882, T98885, Т98888, T98889, T98891, T98892 $2^{\mathrm{DNA}}$, T98894, T98895, T98896, T98897 ${ }^{\mathrm{DNA}}$, T98899 ${ }^{\text {DNA }}$, T98901); 5 males, Aquila Onslow, 17.9 km SW. of Onslow, $21^{\circ} 44^{\prime} 34 \mathrm{~S}, 114^{\circ} 58^{\prime} 45 \mathrm{E}, 29$ October 2008 , dug from burrow, M. Menz (WAM T98881, T98883, T98884, T98887, T98898); 1 male, 27 km NE. of Warrawagine 

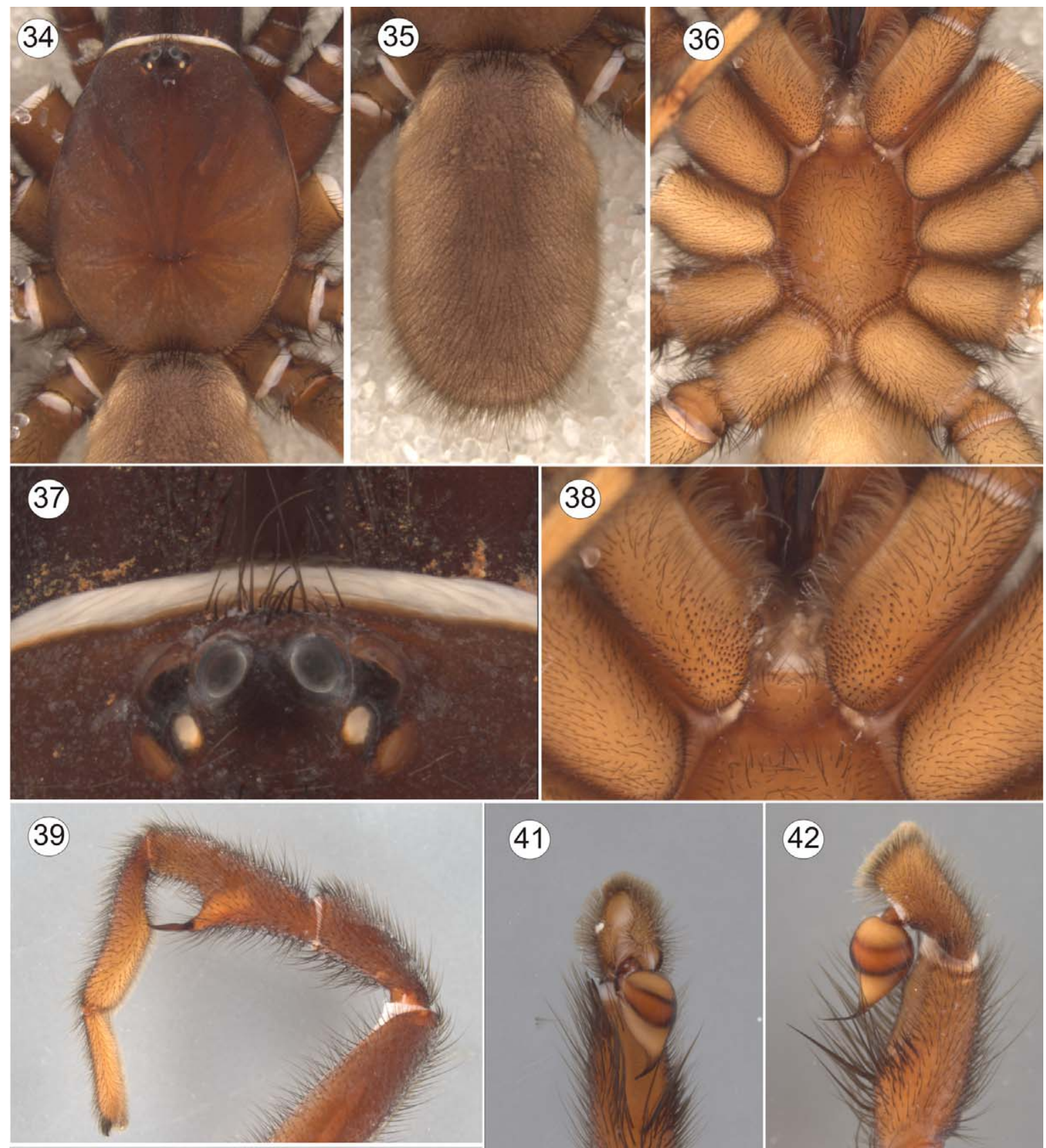

41)
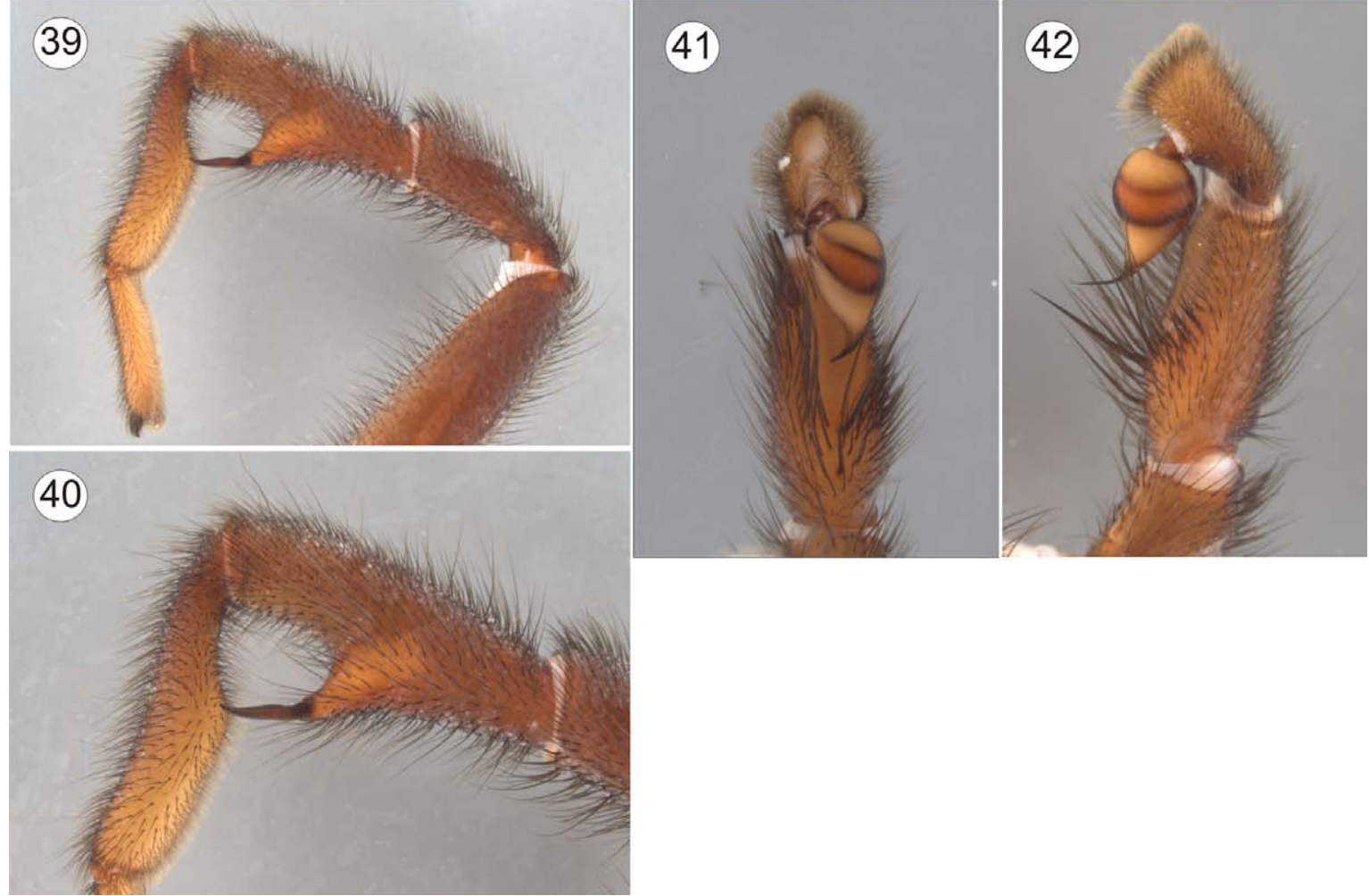

FIGURES 34-42. Aname ellenae n. sp., holotype male (WAM T98890): 34, carapace, dorsal view; 35, abdomen, dorsal view; 36, cephalothorax, ventral view; 37, eye group; 38, maxillae, labium and anterior region of sternum, ventral view; 39, left leg I, retrolateral view; 40, left tibia and metatarsus I, retrolateral view; 41, left pedipalpal tibia and tarsus, ventral view; 42, same, retrolateral view. 
Homestead, site PHYE02, 2042’17S, 12054’04E, 1 July 2005-21 August 2006, ethylene glycol pits, DEC staff (WAM T97306); 1 male, $12.5 \mathrm{~km} \mathrm{~S}$. of Whim Creek Hotel, Pilbara Biological Survey site DRE11B, 20 56'59.6”'S, 117 50'59.6”'E, 13 May 2004-2 May 2005, wet pitfall trap, CALM Pilbara Survey (WAM T97312).

Etymology. This species is named for Ellen Harvey, the senior author's sister.

Diagnosis. Males of A. ellenae resemble A. barrema Raven, 1985, A. distincta Raven, 1985, A. inimica Raven, 1985 and A. marae in the shape of the bulb which gradually tapers to the embolus (Figs 41, 42). The shape and proportions of metatarsus I and tibia I of A. ellenae differ from these species: metatarsus I is much stouter in A. barrema (3.8 x longer than deep), A. distincta (ca. $3.2 \mathrm{x}$ ) and A. inimica (ca. $3.0 \mathrm{x})$ than in A. ellenae (ca. $4.2 \mathrm{x})$ (Fig. 40) and the base of the tibial spur arises abruptly in A. barrema, A. distincta and A. inimica but arises gently in $A$. ellenae (Fig. 40) and A. marae (Fig. 49). Aname ellenae differs from A. marae in details of the pedipalp: the embolus of A. ellenae is much less curved and almost circular in cross-section (Fig. 41); in contrast, the embolus base of A. marae is broadly flattened (Fig. 50). In addition, the pedipalpal tibia has a patch of short strong setae retrolaterally in A. ellenae (Figs 41, 42), which is absent in A. marae (Figs 50, 51). Females of A. ellenae are unknown.

Description. Adult male (based on holotype WAM T98890): medium-sized nemesiid spider (total length 17.0).

Colour: carapace brown with somewhat darker cephalic region (Fig. 34), eye region dark brown (Fig. 37); sternum light brown, darker towards margins, sigilla light brown and labium brown (Fig. 36); abdomen dorsally dark greyish brown, centrally somewhat darker, ventrally very light brown (Fig. 35); chelicerae dark brown; pedipalp segments uniformly brown; leg I brown with metatarsi and tarsi light brown (Fig. 39), legs II-IV light brown.

Carapace: 6.8 long, 5.5 wide; eye group (Fig. 37) 1.2 wide, 0.7 long; fovea slightly procurved (Fig. 34).

Sternum and labium: 3.7 long, 2.5 wide; 2 pairs of very indistinct elongated sigilla in posterior half (Fig. 36); labium wider than long, slightly indented anteriorly (Fig. 38).

Maxillae: with > 100 pin-like cuspules, extending to heel and almost to the centre of maxillae (Fig. 38).

Chelicerae: without rastellum (Fig. 36), promargin with 8 large teeth, retromargin with 5 smaller teeth proximally.

Abdomen: 7.0 long, 4.2 wide (Fig. 35). Four spinnerets, terminal segment of posterior lateral spinnerets digitiform.

Pedipalp: length of femur 3.4, patella 1.8, tibia 1.9, tarsus 1.4. Femur and patella without spines; tibia with 2 long ventral spines, and 3 disto-prolateral spines and patch of 9 shorter spines retrolaterally, tibia with distinct disto-ventral depression without setae (accommodates bulb and embolus) (Figs 41, 42); tarsus terminally blunt (Fig. 41); bulb elongated, globular, tapering into short embolus, embolus ca. 0.5 long, slightly S-curved (Figs 41, 42).

Legs: femur I with 1 dorsal spine in basal half and 2 disto-prolateral spines, patella with 2 prolateral spines, metatarsi and tarsi without spines. Tibia I with large spur and megaspine, metatarsi incrassate (Figs 39, 40). Tarsi and apical half of metatarsi of leg I and II ventrally with entire dense scopulae, tarsi of legs III and IV with divided very weak scopulae. Tarsi, metatarsi and tibiae with numerous dorsal trichobothria of variable length. Paired tarsal claws of legs with two rows of 10-14 teeth each; third tarsal claw reduced and very small.

Leg measurements: length of legs IV >I $>$ II $>$ III. leg I: femur 5.4, patella 3.3, tibia 3.7, metatarsus 3.8, tarsus 2.4, total $=18.6$. Dorsal proximal width of patella $I=1.2$, tibial index $=0.17$, leg I formula $=2.74$. Leg II: femur 4.8, patella 2.6, tibia 2.7 , metatarsus 3.4 , tarsus 2.2 , total $=15.7$. Dorsal proximal width of patella $\mathrm{II}=1.1$, tibial index $=$ 0.21; leg II formula $=2.31$. Leg III: femur 4.2, patella 2.5, tibia 2.6, metatarsus 3.4, tarsus 2.2, total $=14.9$. Dorsal proximal width of patella III $=1.3$, tibial index $=0.26$; leg III formula $=2.19$. Leg IV: femur 5.3, patella 2.9 , tibia 4.2 , metatarsus 4.7, tarsus 2.7 , total $=19.8$. Dorsal proximal width of patella IV $=1.4$, tibial index $=0.20$; leg III formula $=2.91$. Variation: total length $16.2-19.7$, carapace length $6.5-8.0$, carapace width 5.2-6.5 $(n=6)$.

Adult female: unknown.

Distribution. Aname ellenae has been found along the northern coast of the Pilbara, but also along the Fortescue Marsh in the Central Pilbara (Fig. 7); spiders of these apparently separated populations are morphologically indistinguishable; future molecular analyses may shed light on their population genetics and if these populations are reproductively isolated.

Remarks. At the population around Onslow, most male specimens were dug from burrows in October, but were apparently not yet reproductively active. They were possibly waiting for the first seasonal rainfalls to leave their burrows in search of females and therefore appear to be reproductively active early in the wet season. 
Aname marae n. sp.

Figs $8,9,43-51$

Type material. AUSTRALIA: Western Australia: holotype male, Tom Price powerlines, $4 \mathrm{~km} \mathrm{NW}$. of Tom Price, 22 $41^{\prime} 10^{\prime \prime S}, 117^{\circ} 44^{\prime} 56$ ”E, 6 September 2009, dug from burrow, D. Kamien (WAM T98424 ${ }^{\mathrm{DNA}}$ ). Paratypes. 3 males, same data as holotype except 5 September 2009, dug from burrow, D. Kamien (WAM T98427 ${ }^{\text {DNA }}$, T112543, T112544); 3 males, same data except 22 $40^{\prime} 56^{\prime}$ 'S, $117^{\circ} 45^{\prime} 06^{\prime}$ E, 5 September 2009, dug from burrow, D. Kamien (WAM T98428, T98432 $2^{\mathrm{DNA}}$, T98435 ${ }^{\mathrm{DNA}}$ ).

Other material examined. AUSTRALIA: Western Australia: 2 males, Tom Price powerlines, $5 \mathrm{~km} \mathrm{NW}$. of

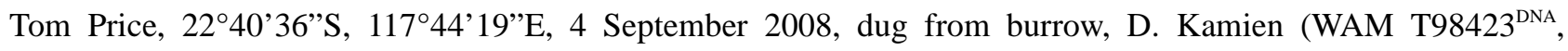

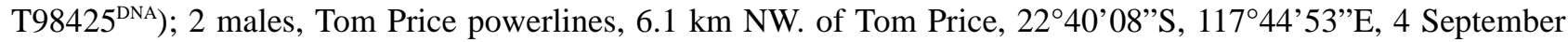
2009, dug from burrow, D. Kamien (WAM T98430 ${ }^{\text {DNA }}$, T112542); 1 male, Tom Price powerlines, $8 \mathrm{~km} \mathrm{NW.} \mathrm{of}$ Tom Price, 22 $2^{\circ} 9^{\prime} 17^{\prime}$ 'S, $117^{\circ} 42^{\prime} 59^{\prime \prime}$ E, 6 September 2009, dug from burrow, D. Kamien (WAM T98436 ${ }^{\mathrm{DNA}}$ ); 5 males, same data except $22^{\circ} 39^{\prime} 28^{\prime \prime} \mathrm{S}, 117^{\circ} 43^{\prime} 01^{\prime} \mathrm{E}$, 6 September 2009, dug from burrow, D. Kamien (WAM T98426 ${ }^{\mathrm{DNA}}, \mathrm{T} 98429^{\mathrm{DNA}}, \mathrm{T} 98431^{\mathrm{DNA}}, \mathrm{T} 98433^{\mathrm{DNA}}, \mathrm{T} 98434^{\mathrm{DNA}}$ ).

Etymology. This species is named for Māra Blosfelds, the senior author's mother.

Diagnosis. Males of A. marae resemble A. barrema Raven, 1985, A. distincta Raven, 1985, A. inimica Raven, 1985 and A. ellenae in the shape of the bulb which tapers gradually to the embolus (Figs 50, 51), but differ in the shape of the embolus which is broadly flattened at the base (Fig. 51). In addition, A. marae males lack the retrolateral patch of short setae on the pedipalpal tibia (Figs 50, 51) that are characteristic for A. ellenae (Figs 41, 42) and also have fewer maxillary cuspules $(<100$ in comparison to $>100)$ which do not extend to the maxillary heel (Fig. 47). Females of $A$. marae are unknown.

Description. Adult male (based on holotype WAM T98424): medium-sized nemesiid spider (total length 13.4).

Colour: carapace reddish-brown with somewhat darker cephalic region (Fig. 43), eye region dark brown (Fig. 46); sternum light brown, darker towards margins, sigilla light brown and labium brown (Fig. 45); abdomen dorsally dark greyish-brown (Fig. 44), ventrally light brown; chelicerae very dark brown; pedipalp segments uniformly brown (Fig. 50); leg I dark reddish-brown with metatarsi and tarsi brown (Fig. 48), legs II-IV brown.

Carapace: 7.2 long, 5.6 wide; eye group (Fig. 46) 1.4 wide, 0.7 long; fovea slightly procurved (Fig. 43).

Sternum and labium: 4.4 long, 2.7 wide; 2 pairs of very indistinct elongated sigilla in posterior half (Fig. 45); labium wider than long, slightly indented anteriorly (Fig. 47).

Maxillae: with < 100 pin-like cuspules, not extending to heel (Fig. 47).

Chelicerae: without rastellum, promargin with 11 irregularly sized teeth, retromargin with 3 small teeth proximally.

Abdomen: 6.7 long, 3.7 wide (Fig. 44). Four spinnerets, terminal segment of posterior lateral spinnerets digitiform.

Pedipalp: length of femur 3.8, patella 2.2, tibia 2.5, tarsus 1.7. Femur with 1 disto-prolateral spine, patella with 2 prolateral spines. Tibia with 2 long ventral spines, and 4 disto-prolateral; with distinct disto-ventral depression without setae (accommodates bulb and embolus) (Fig. 50); tarsus terminally blunt (Fig. 51); bulb elongated, globular, tapering into short embolus (Figs 50,51), embolus ca. 0.8 long, curved and flattened at base, straight and tapering distally (Figs 50, 51).

Legs: femur I with 2 dorsal spines and 2 disto-prolateral spines, patella with 2 prolateral spines, metatarsi and tarsi without spines; tarsi and apical half of metatarsi of leg I and II ventrally with entire dense scopulae, tarsi of legs III and IV with divided very weak scopulae. Tibia I with large spur and megaspine, metatarsi incrassate (Fig. 49). Tarsi, metatarsi and tibiae with numerous dorsal trichobothria of variable length. Paired tarsal claws of legs with two rows of 8-12 teeth each; third tarsal claw reduced and very small.

Leg measurements: length of legs IV >I $>$ II $>$ III. Leg I: femur 5.8, patella 3.5, tibia 4.1, metatarsus 4.2, tarsus 2.5 , total $=20.1$. Dorsal proximal width of patella $\mathrm{I}=1.2$, tibial index $=0.16$, leg I formula $=2.79$. Leg II: femur 5.2 , patella 2.8 , tibia 3.6 , metatarsus 3.8 , tarsus 2.3 , total $=17.7$. Dorsal proximal width of patella $\mathrm{II}=0.9$, tibial index $=0.14$; leg II formula $=2.46$. Leg III: femur 4.4, patella 2.5, tibia 2.7, metatarsus 3.6, tarsus 2.1, total = 15.3. Dorsal proximal width of patella III $=1.0$, tibial index $=0.19$; leg III formula $=2.13$. Leg IV: femur 5.8, patella 3.0, tibia 4.2, metatarsus 5.0, tarsus 2.5, total $=20.5$. Dorsal proximal width of patella IV $=1.1$, tibial index $=0.15$; leg III formula $=2.85$. 


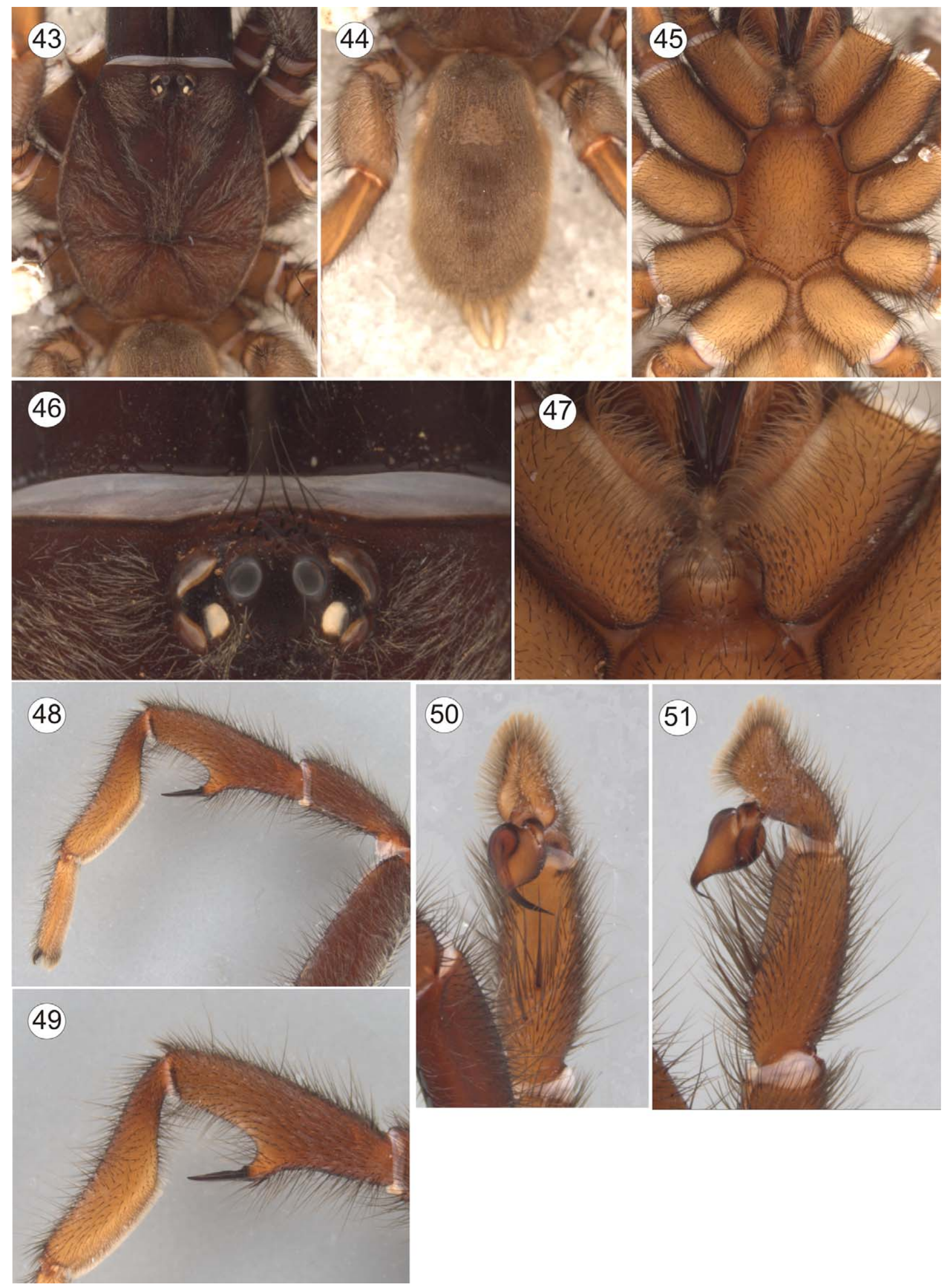

FIGURES 43-51. Aname marae n. sp., holotype male (WAM T98424): 43, carapace, dorsal view; 44, abdomen, dorsal view; 45, cephalothorax, ventral view; 46, eye group; 47, maxillae, labium and anterior region of sternum, ventral view; 48, left leg I, retrolateral view; 49, left tibia and metatarsus I, retrolateral view; 50, left pedipalpal tibia and tarsus, ventral view; 51, same, retrolateral view. 
Variation: total length 14.6-18.1, carapace 6.1-7.6 long, 4.7-5.8 wide $(n=10)$.

Adult female: unknown.

Distribution. Aname marae has only been found near the township of Tom Price in the central Pilbara region of Western Australia (Fig. 8).

Remarks. As for A. ellenae (see above), most males of A. marae were dug from burrows before the beginning of the wet season (in September) and may have waited there for the first seasonal rainfalls before searching for females.

\section{Acknowledgements}

This project was part of the sanofi-aventis BioGeneius Challenge of Western Australia and the sanofi-aventis International BioGeneius Challenge. We thank the Western Australian Department of Industry and Resources, and particularly Janelle Barnes and Jocelyn Bailey, for their support and the opportunity to present the results of this study at the 2010 Biotechnology Industry Organization's Annual International Convention in Chicago, U.S.A. We also thank Mr Warwick Mathews and Mr Michael Morgan from Shenton College for their support of the project, and for Mr Mathews' suggestion that FSBH enter the BioGeneius Challenge. Anna Leung (Phoenix Environmental Sciences) took the digital images of the female of $A$. mellosa. We are also very grateful to staff from Biota Environmental Sciences, Outback Ecology, the School of Animal Biology at the University of Western Australia for the use of molecular laboratory facilities, and the Australian Museum Business Service for supplying some of the specimens used in this study, and to Roy Teale for his cheerful support and for supplying the images of the burrow of $A$. mellosa.

\section{References}

Barrett, R.D.H. \& Hebert, P.D.N. (2005) Identifying spiders through DNA barcodes. Canadian Journal of Zoology, 83, 481-491.

Brito, P.H. \& Edwards, S.V. (2009) Multilocus phylogeography and phylogenetics using sequence-based markers. Genetica, 135, 439-455.

Brown, R.W. (1956) Composition of Scientific Words. Smithsonian Institution Press, Washington, D.C., 882 pp.

Coyle, F.A. (1971) Systematics and natural history of the mygalomorph spider genus Antrodiaetus and related genera (Araneae: Antrodiaetidae). Bulletin of the Museum of Comparative Zoology, 141, 269-402.

De Queiroz, K. (2007) Species concepts and species delimitation. Systematic Biology, 56, 879-886.

Donoghue, M.J. (1985) A critique to the Biological Species Concept and recommendations for a phylogenetic alternative. The Bryologist, 88, 172-181.

Durrant, B.J., Harvey, M.S., Framenau, V.W., Ott, R. \& Waldock, J.M. (2010) Patterns in the composition of ground-dwelling spider communities in the Pilbara bioregion, Western Australia. Records of the Western Australian Museum, Supplement, 78, 185-204.

Felsenstein, J. (1985) Confidence limits on phylogenies: an approach using the bootstrap. Evolution, 39, $783-791$.

Folmer, O., Black, M., Hoeh, W., Lutz, R. \& Vrijenhoek, R.C. (1994) DNA primers for amplification of mitochondrial cytochrome c oxidase subunit I from diverse metazoan invertebrates. Molecular Marine Biology and Biotechnology, 3, 294-299.

Goloboff, P.A. (1993) A reanalysis of mygalomorph spider families (Araneae). American Museum Novitates, 3056, 1-32.

Goloboff, P.A. (1995) A revision of the South American spiders of the family Nemesiidae (Araneae, Mygalomorphae). Part I: Species from Peru, Chile, Argentina, and Uruguay. Bulletin of the American Museum of Natural History, 224, 1-189.

Harvey, M.S. (2002) Short-range endemism in the Australian fauna: some examples from non-marine environments. Invertebrate Systematics, 16, 555-570.

Harvey, M.S., Rix, M.G., Framenau, V.W., Hamilton, Z.R., Johnson, M.S., Teale, R.J., Humphreys, G. \& Humphreys, W.F. (2011) Protecting the innocent: studying short-range endemic taxa enhances conservation outcomes. Invertebrate Systematics, 25, 1-10.

Hebert, P.D.N., Cywinska, A., Ball, S.L. \& deWaard, J.R. (2003) Biological identifications through DNA barcodes. Proceedings of the Royal Society of London. Series B: Biological Sciences, 270, 313-321.

Hedin, M. \& Bond, J.E. (2006) Molecular phylogenetics of the spider infraorder Mygalomorphae using nuclear rRNA genes (18S and 28S): conflict and agreement with the current system of classification. Molecular Phylogenetics and Evolution, 41, 454-471.

Hogg, H.R. (1901) On Australian and New Zealand spiders of the suborder Mygalomorphae. Proceedings of the Zoological 
Society of London, 1901, 218-279.

Hogg, H.R. (1902) On some additions to the Australian spiders of the suborder Mygalomorphae. Proceedings of the Zoological Society of London, 1902, 121-142.

Koch, L. (1873) Die Arachniden Australiens, nach der Natur beschrieben und abgebildet. Bauer und Raspe, Nürnberg, vol. 1, pp. 369-472.

Koch, L. (1875) Die Arachniden Australiens, nach der Natur beschrieben und abgebildet. Bauer und Raspe, Nürnberg, vol. 1, pp. 577-740.

Main, B.Y. (1954) Spiders and Opiliones. Part 6 of the Archipelago of the Recherche. Australian Geographical Society Reports, 1, 37-53.

Main, B.Y. (1982a) Further studies on the systematics of Australian Diplurinae (Araneae: Mygalomorphae, Dipluridae): the taxonomic status of Proshermacha Simon and Chenistonia tepperi Hogg. Australian Entomological Magazine, 8, 83-88.

Main, B.Y. (1982b) Notes on the revised taxonomic position of the black wishbone spider Dekana diversicolor Hogg (Mygalomorphae: Dipluridae). Journal of the Royal Society of Western Australia, 65, 25-29.

Main, B.Y. (1983) Further studies on the systematics of Australian Diplurinae (Chelicerata: Mygalomorphae: Dipluridae): two new genera from southwestern Australia. Journal of Natural History, 17, 923-949.

Main, B.Y. (1985) Mygalomorphae. In: Walton, D.W. (Ed.) Zoological catalogue of Australia. Australian Government Publishing Service, Canberra, pp. 1-48.

Main, B.Y. (1986) Further studies on the systematics of Australian Diplurinae (Araneae: Mygalomorphae: Dipluridae): a new genus from south-western Australia. Records of the Western Australian Museum, 12, 395-402.

Main, B.Y. (1994) Biosystematics of Australian mygalomorph spiders: description of a new species of Aname and its aerial tube (Araneae: Nemesiidae). Journal of the Royal Society of Western Australia, 77, 65-69.

Main, B.Y., Sampey, A. \& West, P.L.J. (2000) Mygalomorph spiders of the southern Carnarvon Basin, Western Australia. Records of the Western Australian Museum, Supplement, 61, 281-293.

McKenzie, N.L., van Leeuwen, S. \& Pinder, A.M. (2009) Introduction to the Pilbara Biodiversity Survey, 2002-2007. Records of the Western Australian Museum, Supplement, 78, 3-89.

Nixon, K.C. \& Wheeler, Q.D. (1990) An amplification of the phylogenetic species concept. Cladistics, 6, $211-223$.

Petrunkevitch, A. (1942) A study of amber spiders. Transactions of the Connecticut Academy of Arts and Sciences, 34, $119-464$.

Platnick, N.I. (2012) The World Spider Catalog, Version 12.5, http://research.amnh.org/iz/spiders/catalog/INTRO1.html. Accessed 11 March 2012. American Museum of Natural History, New York.

Posada, D. \& Crandall, K.A. (1998) Modeltest: testing the model of DNA substitution. Bioinformatics, 14, 817-818.

Prendini, L. (2005) Comment on "Identifying spiders through DNA barcodes". Canadian Journal of Zoology, 83, $498-504$.

Rainbow, W.J. (1911) A census of Australian Araneidae. Records of the Australian Museum, 9, 107-319.

Rainbow, W.J. \& Pulleine, R.H. (1918) Australian trap-door spiders. Records of the Australian Museum, 12, 81-169.

Raven, R.J. (1981) A review of the Australian genera of the mygalomorph spider subfamily Diplurinae (Dipluridae: Chelicerata). Australian Journal of Zoology, 29, 321-363.

Raven, R.J. (1984) A revision of the Aname maculata species group (Dipluridae, Araneae) with notes on biogeography. Journal of Arachnology, 12, 177-193.

Raven, R.J. (1985a) A revision of the Aname pallida species-group in northern Australia (Anaminae: Nemesiidae: Araneae). Australian Journal of Zoology, 33, 377-409.

Raven, R.J. (1985b) The spider infraorder Mygalomorphae (Araneae): cladistics and systematics. Bulletin of the American Museum of Natural History, 182, 1-180.

Raven, R.J. (2000) Taxonomica Araneae I: Barychelidae, Theraphosidae, Nemesiidae and Dipluridae (Araneae). Memoirs of the Queensland Museum, 45, 569-575.

Ronquist, F. \& Huelsenbeck, J.P. (2003) MRBAYES 3: Bayesian phylogenetic inference under mixed models. Bioinformatics, $19,1572-1574$.

Rowling, J.K. (1998) Harry Potter and the Chamber of Secrets. Bloomsbury, London, 251 pp.

Schmidt, G.E.W. (2000) The mystery of Selenocosmia huwena Wang, Peng \& Xie, 1993, a tarantula from China. Journal of the British Tarantula Society, 15, 77-79.

Simon, E. (1908) Araneae, $1^{\text {re }}$ partie. In: Michaelsen, W. \& Hartmeyer, R. (Eds.) Die Fauna Südwest-Australiens. Gustav Fischer, Jena, pp. 359-446.

Swofford, D.L. (2002) PAUP*. Phylogenetic Analysis Using Parsimony (*and Other Methods). Sinauer Associates, Sunderland, $M A$

Thompson, J.D., Gibson, T.J., Plewniak, F., Jeanmougin, F. \& Higgins, D.G. (1997) The CLUSTAL_X windows interface: flexible strategies for multiple sequence alignment aided by quality analysis tools. Nucleic Acids Research, 25, 4876-4882.

Wang, J.F., Peng, X.J. \& Xie, L.P. (1993) One new species of the genus Selenocosmia from south China (Araneae, Theraphosidae). Acta Scientiarum Naturalium Universitatis Normalis Hunanensis, 16, 72-74.

Zhu, M.S. \& Zhang, R. (2008) Revision of the theraphosid spiders from China (Araneae: Mygalomorphae). Journal of Arachnology, 36, 425-447. 